Sociologie et sociétés

SOCIOLOGIE

ETSOCIÉTÉS

\title{
Le spectacle du monde
}

Nouvelles formes du cosmopolitisme et espaces-temps

fracturés des métropoles contemporaines

\section{The Global Spectacle}

\section{New Forms of the Cosmopolitanism and Space-Time Cleavages of the Contemporary Metropolis}

\section{Jean-François Côté}

Volume 37, numéro 1, printemps 2005

Le spectacle des villes

The Spectacle of Cities

URI : https://id.erudit.org/iderudit/012285ar

DOI : https://doi.org/10.7202/012285ar

Aller au sommaire du numéro

Éditeur(s)

Les Presses de l'Université de Montréal

ISSN

0038-030X (imprimé)

1492-1375 (numérique)

Découvrir la revue

Citer cet article

Côté, J.-F. (2005). Le spectacle du monde : nouvelles formes du cosmopolitisme et espaces-temps fracturés des métropoles contemporaines. Sociologie et sociétés, 37(1), 231-260. https://doi.org/10.7202/012285ar

\section{Résumé de l'article}

La définition du cosmopolitisme au sein de la philosophie politique moderne situait son développement dans une perspective à la fois théologique et utopique ; par rapport à cela, le développement des villes-métropoles contemporaines a établi des liens directs avec une nouvelle conception du cosmopolitisme. Cette conception est désormais directement liée au phénomène métropolitain dans ce que celui-ci réfléchit les conditions d'existence précises et possibles du cosmopolitisme. L'ordre politique universel qui apparaît ainsi dans les villes-métropoles est cependant traversé de contradictions importantes, qui révèlent les fractures spatio-temporelles présentes dans cette définition du cosmopolitisme contemporain. Ce sont ces tensions, affrontements et contradictions qui doivent toutefois guider l'idée que l'on se fait du cosmopolitisme, puisque c'est là que se donne à voir réellement le spectacle du monde, soit un ensemble de phénomènes qui se manifestent en condensé dans l'expérience des villes. L'article prend la mesure de ces transformations contemporaines du cosmopolitisme à travers la perception des villes-métropoles.
Tous droits réservés @ Les Presses de l'Université de Montréal, 2005
Ce document est protégé par la loi sur le droit d'auteur. L’utilisation des services d’Érudit (y compris la reproduction) est assujettie à sa politique d'utilisation que vous pouvez consulter en ligne.

https://apropos.erudit.org/fr/usagers/politique-dutilisation/ 


\section{Le spectacle du monde \\ Nouvelles formes du cosmopolitisme et espaces-temps fracturés des métropoles contemporaines}

\section{JEAN-FRANÇOIS CÔTÉ}

Département de sociologie

Université du Québec à Montréal

C.P. 8888, Succursale Centre-ville

Montréal (Québec), Canada $\mathrm{H}_{3} \mathrm{C}_{3} \mathrm{P} 8$

Courriel : cote.jean-francois@uqam.ca

\section{$\mathrm{L}$}

ES RAPPORTS QUI EXISTENT ENTRE LES MÉTROPOLES ET LE COSMOPOLITISME SONt res-

tés, jusqu'à récemment, l'objet de peu d'interprétations sociologiques; cela peut paraître très surprenant, si l'on tient compte du fait qu'il va pratiquement de soi pour le sens commun que, s'il existe une forme de cosmopolitisme dans la société contemporaine, c'est dans le contexte des grandes villes, des métropoles, que l'on en trouve l'expression particulière. Mais cette idée du sens commun n'est pas, elle non plus, tellement développée en ce qui a trait justement à l'appréhension plus précise de ces formes du cosmopolitisme, au-delà de leurs déterminations très générales. On peut donc se permettre de tenter d'aller dans ce sens, au-delà du sens commun et de son approximation, en s'interrogeant plus spécifiquement sur ce qui définit, aujourd'hui, les rapports entre les formes - et surtout les formes nouvelles - du cosmopolitisme et les métropoles contemporaines. Je ferai ici cet effort, en me penchant plus spécifiquement sur la manière par laquelle le «spectacle du monde» qui se manifeste dans les villes-métropoles contemporaines nous informe sur les formes nouvelles du cosmopolitisme, en prenant appui non pas spécifiquement sur la dimension proprement «spectaculaire» des villes, sur laquelle on a tant insisté ces dernières années dans la littérature sociologique et les études urbaines, mais bien, et plus généralement, sur la façon dont le monde se "donne à voir» aujourd'hui à travers 
l'existence citadine métropolitaine ${ }^{1}$. C'est de cette manière que j'entends montrer comment les villes-métropoles deviennent des révélateurs d'un ordre du monde universel, ce qui correspond à une des exigences fondamentales du cosmopolitisme, dont la représentation et la conceptualisation étaient devenues éminemment problématiques au fur et à mesure que se réalisaient les développements de la société contemporaine, semblant l'emporter bien au-delà de ses déterminations principales héritées de la modernité bourgeoise européenne. Je montrerai ainsi que cette représentation et cette conceptualisation se donnent selon des acceptions spatiotemporelles disjointes qui se réfléchissent ainsi dans des formes d'expressions sociales et des phénomènes culturels qui nous forcent à envisager une redéfinition sociohistorique de leurs contenus tels qu'ils se manifestent dans le spectacle des villes-métropoles contemporaines.

\section{Villes-métropoles et vie politique}

Le problème de l'étude de ces rapports entre villes-métropoles et cosmopolitisme provient sans doute en bonne partie du fait que la définition des conditions politiques de l'existence sociale échappe très largement aux villes et aux métropoles seules, pour se rattacher plutôt, d'un côté, aux formes de l'État-nation contemporain - une situation qui, comme on le sait, est toute différente de la façon dont la cité-État européenne s'est établie, et différente aussi de la façon dont les « communes» de la modernité conféraient aux «bourgeois», c'est-à-dire aux habitants des «bourgs », cette qualité de « citoyen » (dont le terme anglais «citizen» rend encore, dans son sens littéral, cette consonance étroite d'appartenance à la ville, et dont Max Weber avait saisi le sens d'implication étroite au devoir citoyen qu'il impliquait dans le serment de « conjuration» au sein des communes bourgeoises à l'aube de la modernité [Weber, 1982, p. 64-84]). Aujourd'hui, et en fonction de son inclusion dans les termes de la citoyenneté nationale, tout individu est comme on le sait « citoyen », sans que cela souligne son appartenance à l'existence strictement urbaine, encore moins métropolitaine; davantage, et en fonction de l'énoncé de la Déclaration universelle des droits de l'homme, tout individu est considéré, au moins formellement, dans ses dispositions politiques universelles, en tant que « citoyen du monde», quel que soit par ailleurs son positionnement effectif au sein de l'ordre social. D’un autre côté, les rapports internationaux qui structurent les relations entre États-nations donnent une certaine configuration à l'ordre cosmopolitique contemporain - mais c'est alors en fonction d'une définition qui ne rejoindrait plus que de très loin, en apparence du moins, la définition des villes, ou en

1. Ce faisant, je rends la notion de «spectacle » à son acception large, qui la distingue du «spectaculaire » au sens strict, de la même manière dont le "theatrum mundi», soit ce qu'on appelle métaphoriquement le «théâtre du monde», se distingue du « théâtre» proprement dit, c'est-à-dire de cet espace restreint de représentation ayant une vocation précisément artistique; la dimension « esthétique» du spectacle des villes-métropoles est ainsi invoquée plus largement, et en dehors de la destination expressément « spectaculaire » que lui ont prêtée par exemple Saskia Sassen (1998), Sharon Zukin (1995) ou David Harvey (1992), dans leurs évaluations respectives de la culture contemporaine des villes. «Spectacle» (spectaculum) se rapproche donc ici de sa définition simple de "ce qui s'offre au regard ", en conservant toutefois cette propension "spéculative», cette capacité de réfléchir ou de spéculer (speculari), qui lui est étymologiquement liée. La réflexion conceptuelle prend alors appui sur la perception esthétique au sens large du terme. 
tout cas, qui ne semblerait pas lui appartenir ni lui être destinée en propre, non plus que la faire participer directement au cosmopolitisme d'ensemble (particulièrement lorsque celui-ci se donne dans les formes des organisations internationales telles que l'oNu, l'unEsco, l'OMc, etc., entités qui restent relativement abstraites, c'est-à-dire absentes, en apparence du moins, de la vie quotidienne de la plupart des individus, de même que ces entités sont relativement indépendantes de leur localisation en un point spécifique du monde, c'està-dire dans une ville particulière ${ }^{2}$ ). Si la « ville-métropole » a pu ainsi signifier un point de référence central dans la définition de l'ordre du monde, le monde politique contemporain prend davantage appui sur une répartition «poly-centrée» de sa composition justement «inter-nationale», puisque New York, Paris, Genève ou Montréal peuvent en être les hôtes, en même temps que la « localisation » du pouvoir politique s'est faite elle aussi plus diffuse dans le cours même de sa définition mondiale (Ferrarotti, 1995).

Du strict point de vue des villes, et en particulier des métropoles, il ne semble pas non plus que la question du cosmopolitisme puisse être liée de quelque façon aux activités principales qui les définissent - dans la mesure où, justement, ces activités très localisées sont plutôt à caractère économique, social ou même culturel, ce qui renvoie en apparence la dimension politique de l'existence métropolitaine à l'arrière-plan de son existence réelle. Et pourtant, on aurait de grandes difficultés à appréhender la question du cosmopolitisme en dehors d'une référence directe aux villes-métropoles. Une des premières tentatives sociologiques d'appréhension du rapport entre métropole et cosmopolitisme apparaît d'ailleurs significative de ce point de vue, puisqu'elle lie de manière très étroite la signification de la métropole et la signification de l'esprit contemporain dans sa formation individuelle. En effet, dans son texte "Métropoles et mentalité », devenu classique depuis sa parution originale en 1903, Georg Simmel voit très bien comment la dynamique cosmopolitique apparait appartenir en propre au caractère de la ville-métropole, et unir son destin à celui de la personnalité individuelle, lorsqu'il écrit:

Ce n'est pas seulement la grandeur effective du territoire et du nombre d'hommes qui fait de la grande ville le siège de la liberté personnelle, interne et externe, bien que, selon l'histoire mondiale, celle-ci soit en corrélation avec l'accroissement du milieu [Kreis]; mais c'est en allant au-delà de cette expansion visible que les grandes villes ont été aussi le siège du cosmopolitisme. (...) Le caractère le plus significatif de la grande ville tient à cette extension fonctionnelle qui dépasse ses frontières physiques: cette activité produit un effet de retour et donne à la vie de la grande ville du poids, de l'importance, de la responsabilité. De même qu'un homme ne se limite pas aux frontières de son corps ou du territoire qu'il remplit immédiatement de son activité, mais seulement à la somme des actions qui s'étendent à partir de lui dans le temps et dans l'espace, de même également une ville ne subsiste que de la somme des actions qui étendent son empire au-delà de ses confins immédiats. C’est là seulement la dimension véritable où son être s'exprime. (Simmel, 1984, p. 71-72)

2. Cela n'exclut pas par ailleurs que des rapports plus directs se manifestent à cet égard, comme en témoignent le support et l'attention de l'unEsco dans la revalorisation du patrimoine architectural de La Havane, par exemple - voir à ce sujet Alain Caron (1999). 
Lorsqu'il situe la ville-métropole comme centre du rayonnement du cosmopolitisme, Simmel semble oblitérer le fait qu'il en est ainsi parce que la ville-métropole réfléchit en elle-même un mouvement qui la dépasse, et même, d'une certaine façon, qui lui préexiste ; en effet, ce n'est pas la ville-métropole en soi qui est à l'origine de ce mouvement, bien qu'elle s'en fasse le relais, et pour tout dire, qu'elle lui fournisse la matière de sa manifestation. Au contraire, la ville-métropole devient telle parce qu'elle parvient à réfléchir en elle-même un mouvement qui est à la mesure de ce qu'elle pourra représenter ou, en d'autres termes, elle acquiert une telle signification dans la mesure où elle condense en elle-même un mouvement qui lui donnera en retour une extension spatiotemporelle déterminée ${ }^{3}$. Et en dépit d'une absence de spécification directe du caractère politique de la ville-métropole chez Simmel, je voudrais montrer ici que c'est bien l'expérience élargie du politique, atteignant cependant une dimension mondiale, telle qu'elle apparaît particulièrement dans son contexte métropolitain, qui donne un sens aux nouvelles formes du cosmopolitisme dans la société contemporaine. Pour ce faire, je vais procéder d'abord à un bref examen de la signification que le cosmopolitisme avait dans le contexte de la modernité bourgeoise européenne, pour montrer ensuite comment son évolution actuelle ou récente - puisque je parlerai essentiellement des deux cents dernières années - révèle ce lien obligé avec la ville-métropole. Ainsi, et jusqu'à un certain point à l'encontre de ce que soulignait Simmel, je voudrais proposer que cette extension politique de la ville-métropole se compare à l'extension à laquelle peut prétendre la subjectivité individuelle dès lors qu'elle se rattache à une définition qui n'est plus étroitement définie par ses limites spatiotemporelles urbaines, ni même nationales, mais qu'elle atteint une résonance potentiellement internationale, ou universelle - ce qui se produit justement dans l'histoire contemporaine; pour autant que la ville-métropole possède en effet cette capacité de manifester en elle la mesure de ce mouvement, elle réfléchit alors les conditions du cosmopolitisme contemporain ${ }^{4}$. C'est dans ce sens que je parlerai enfin des nouvelles formes de cosmopolitisme en rapport aux nouveaux espaces-temps des villes-métropoles contemporaines, en montrant surtout leurs fractures, de même que les exigences de médiations symboliques ainsi mises au jour.

3. Ce qui n'était évidemment pas le cas de la «ville-métropole» de l'Antiquité; lorsque Athènes ou Rome, par exemple, apparaissaient en elles-mêmes comme les centres de décision politique, c'est parce qu'elles parvenaient à concentrer pour ainsi dire « dans leurs murs » la signification complète du statut (politique, économique, militaire) qui leur conférait cette signification, et donc que tout ce mouvement partait de la ville (Max Weber, 1982, p. 85-125). Voir aussi à ce sujet de Coulanges (1984). La situation contemporaine est évidemment complètement différente.

4. Sur ce plan, on voit donc que la signification du statut cosmopolitique du «citoyen » correspond, au moins formellement, au statut de «métropole» tel que l'entend Simmel. Conséquemment, le statut de «citoyen du monde» contemporain ne peut plus correspondre seulement à l'individu d'une «ville-métropole», bien que ce dernier en devienne le représentant exemplaire. 


\section{COSMOPOlitisme MOderne ET « POSTMOderne»: PASSAGE PAR BerLin}

Le cosmopolitisme moderne trouve sans doute sa définition classique et achevée chez Emmanuel Kant, dans ses deux essais tardifs, Idée d'une histoire universelle d'un point de vue cosmopolitique, daté de 1784, où sont exprimées les neuf propositions en vue de l'unification politique des États dans le monde, ainsi que les trois « articles définitifs », présentés dans le texte Vers la paix perpétuelle, daté de 1795. Dans ces deux essais, Kant appréhende la question du cosmopolitisme en fonction des conditions permettant d'entrevoir le développement d'une fédération d'États nationaux fondés sur le droit par le biais de constitutions républicaines, ceci devant mener au développement d'une paix universelle et durable, soit d'un ordre politique s'appliquant à l'humanité tout entière. Ainsi s'exprime pour Kant, dans la Septième proposition de son Idée d'une histoire universelle d'un point de vue cosmopolitique, la nécessité de:

... sortir de l'absence de loi propre aux sauvages pour entrer dans une Société des Nations dans laquelle chaque État, même le plus petit, pourrait attendre sa sécurité et ses droits, non de sa propre force ou de sa propre appréciation du droit, mais uniquement de cette grande Société des Nations (Fœdus Amphictyonum), c'est-à-dire d'une force unie et de la décision légale de la volonté unifiée. (Kant, réédition de 1985, p. 487)

Cette expression ultime de la pensée politique moderne que l'on trouve chez Kant doit nous frapper aujourd'hui autant par son prodigieux pouvoir d'anticipation que par sa propension toujours relativement utopique, comme le rappellent sans cesse les événements politiques et militaires du monde contemporain, et comme l'a rappelé également Jürgen Habermas (2000), puisqu'elle constitue en quelque sorte l'horizon du développement historique des relations internationales depuis plus de deux siècles; dans cette évolution historique ainsi que dans le sens qu'on lui attribue, en effet, c'est l'ensemble du projet d'émancipation de la modernité qui trouverait en quelque sorte son terme ultime, c'està-dire sa réussite ou son échec. Et Kant donne même une expression qui semble s'appliquer au plus près à notre condition actuelle lorsqu'il écrit, dans l'explicitation de sa Huitième proposition :

Bien que ce corps politique n'existe encore pour l'instant qu'à l'état d'ébauche très grossière, un sentiment se fait déjà pour ainsi dire jour chez tous ses membres dont chacun tient à la conservation du tout : et cela donne l'espérance qu'après maintes révolutions survenues dans cette transformation parviendra finalement un jour à s'établir ce que la nature a pour dessein suprême d'établir, à savoir une situation cosmopolitique universelle comme foyer au sein duquel se développeraient toutes les dispositions originelles de l'espèce humaine. (Kant, 1985 , p. 492, italiques dans l'original)

La tâche du cosmopolitisme, on le voit, est chez Kant énorme et palpitante. Or, ce qui ne peut manquer de nous frapper dans cette dernière citation, c'est bien sûr la question de l'inéluctabilité «naturelle» du processus de constitution de la situation cosmopolitique, une «nature » confondue chez Kant à son terme avec les lois de la « Providence», la téléologie historique se rapportant ici à une théologie, ou plus exactement, à l'actualisation 
moderne de la théologie chrétienne ${ }^{5}$. Bien que n'étant pas «idéaliste» au point d'oublier que les rapports humains s'établissent fondamentalement au sein de cette «insociable sociabilité», comme il l'appelle, Kant n'en demeurait pas moins peut-être trop "optimiste» dans son appréhension du développement socio-historique, comme en témoignera éloquemment, et dans sa suite immédiate, la critique que Hegel, puis Marx, feront de la situation d'évolution historique du monde. Chez Hegel, en effet, c'est davantage dans le réalisme de la guerre et de l'impérialisme que se définit cette évolution, comme il l'écrit dans ses Principes de la philosophie du droit ainsi que dans ses Leçons sur la philosophie de l'histoire, alors que chez Marx, c'est l'horizon dernier de la révolution prolétarienne qui en détermine comme on le sait le cours ultime ${ }^{6}$. Sans aller beaucoup plus loin dans l'appréhension des formes du cosmopolitisme héritées de la philosophie politique moderne chez Kant, et de ses suites, voire de ses dépassements, chez Hegel et chez Marx, on peut aisément constater que l'évolution socio-historique elle-même s'est chargée, aux $\mathrm{XIX}^{\mathrm{e}}$ et $\mathrm{XX}^{\mathrm{e}}$ siècles, de faire jouer dans un certain sens les diverses catégories impliquées ici. Une des expressions les plus audacieuses de cette évolution provient incidemment de Randolph Bourne, cet intellectuel étatsunien marginal, qui, dans son texte Transnational America, publié en 1916, appliquait aux États-Unis du xx ${ }^{\mathrm{e}}$ siècle la capacité de déterminer cette nouvelle forme de cosmopolitisme, en écrivant:

Dans un monde qui a rêvé d'internationalisme, nous constatons que nous avons tous inconsciemment contribué à construire la première nation internationale. Les voix qui ont appelé à un

5. Dans l'explicitation de sa Neuvième proposition, qui réalise l'actualisation moderne de la théologie chrétienne, Kant écrit ainsi : «Une telle justification de la nature - ou mieux, de la Providence - est un motif non négligeable pour choisir un point de vue particulier dans la contemplation du monde. Car à quoi bon vanter la magnificence et la sagesse de la création dans le domaine de la nature d'où la raison est absente, à quoi bon recommander cette contemplation si la partie de la vaste scène de la sagesse suprême qui précisément contient le but de tout le reste - à savoir l'histoire de l'espèce humaine - doit demeurer une éternelle objection dont la vue nous contraint, à contrecour, à détourner les yeux de ce spectacle et nous conduit, désespérés d'y jamais rencontrer un dessein rationnel parfait, à n'espérer celui-ci que dans un autre monde.» (Kant, 1985, p. 494, italiques dans l'original) On sait par ailleurs que toute l'éthique kantienne prend appui sur la rationalisation de la notion de "bonne volonté», ce qui appuie fondamentalement son ancrage chrétien. Je fais également remarquer ici en passant la manière par laquelle Kant appréhende justement le «spectacle du monde », qui tient, dans ses propres termes, à cette capacité de "contemplation de l'histoire» assistée par un "dessein rationnel parfait».

6. Du point de vue hégélien, on devrait retenir que c'est en fait l'« esprit du monde» (Weltgeist) qui apparaît comme la scène de représentation sur laquelle s'animent les différents événements, et que ceux-ci impliquent avant tout des États; ce mouvement de l'esprit, cette inquiétude, telle qu'elle prend forme dans la liberté politique réalisée dans les termes de l'État-nation, est en effet réellement celle qui conduit pour Hegel le développement historique, déterminé également de manière téléologique par le concept de liberté. C’est entre autres la raison pour laquelle Hegel (1998, p. 279) s'oppose à l'idée de cosmopolitisme en tant que moralité citoyenne; pour lui, cette dernière ne peut avoir qu'un ancrage national. Marx retiendra plutôt comme on le sait l'idée du conflit de classes comme moteur du développement historique, en critiquant d'un côté l'étroitesse du nationalisme bourgeois, et en s'appuyant de l'autre sur la définition de l'«être social» au cour des sociétés humaines portées par le développement téléologique de l'idée du communisme (à la fois comme condition historique primitive et comme finalité de leur développement) ; ses propres efforts de représentation d'un ordre politique universel porteront alors sur la mise en évidence de la lutte des classes au sein du processus social (lutte ramenée cependant la plupart du temps à sa rationalisation plus abstraite dans les termes économiques de l'opposition capital-travail). 
nationalisme étroit et orgueilleux sur le modèle européen ont échoué. Le temps et les tendances nous ont éloignés de plus en plus de cet idéal qui nous avait été si vaillamment et librement légué. Ce que nous avons plutôt réalisé est une fédération cosmopolitique de colonies nationales, de cultures étrangères, desquelles l'aiguillon de la compétition dévastatrice a été éliminé. L’Amérique [i.e. les États-Unis] représente déjà la fédération du monde en miniature, le continent où pour la première fois de l'histoire s'est réalisé cet espoir miraculeux, cette cohabitation pacifique, côte à côte, tout en préservant en substance chacun de ses caractères, de ce peuple le plus hétérogène sous le soleil $7^{7}$. (Traduction, Bourne, 1964, p. 117, je souligne)

Des guerres impérialistes européennes au projet de la Société des Nations, des deux guerres mondiales à la guerre froide, de l'Organisation des Nations Unies à la Pax americana, l'évolution sociohistorique contemporaine a bien imprimé dans nos consciences les possibilités et les limites du développement du cosmopolitisme - du moins dans sa version moderne. À l'unification présumée de la culture sous l'égide de l'État-nation, encore présente chez Kant et chez Hegel, mais déjà très critiquée par Marx en raison de son caractère étroitement intéressé et bourgeois, a succédé un intense mélange des cultures nationales résultant des avatars des relations internationales contemporaines, sous leurs formes politiques, économiques et sociales, marqué par la présence du nouvel impérialisme étatsunien, et c'est ce qui forme l'empreinte la plus sensible de nos sociétés actuelles dans la mesure du moins où, comme le soulignait Bourne, l' " hétérogénéité » des populations succède à une simple subsomption des individus sous une acception culturelle nationale. L'horizon téléologique rationnel de la modernité, autant dans son contenu utopique que théologique, s'est ainsi d'abord refermé sur ses propres limites, puis rouvert par la suite sur les perspectives de l'évolution sociohistorique concrète des deux derniers siècles. Et c'est l'interprétation de cette réouverture qui conduit l'interrogation au sujet du cosmopolitisme contemporain, tel qu'il va précisément se réfléchir dans les villesmétropoles.

Le cosmopolitisme moderne avait donc culminé dans la vision d'une expérience sociohistorique associée au triomphe de la bourgeoisie européenne, et il ne s'ouvrait alors en fait qu'à la perception d'un monde mené par une élite nationale sublimant sa propre transcendance dans les réalisations d'une domination universelle - elle-même objet de rivalités

7. À ceci, Bourne ajoutait: «Only America, by reason of the unique liberty of opportunity and traditional isolation for which she seems to stand, can lead in this cosmopolitan entreprise. Only the American - and in this category I include the migratory alien who has lived with us and caught the pionneer spirit and a sense of new social vistas - has the chance to become that citizen of the world. America is coming to be, not a nationality but a transnationality, a weaving back and forth, with the other lands, of many threads of sizes and colors. » (p. 121) L'expression de cette «trans-nationalité étatsunienne», chez Bourne, contredit la définition que donnait Kant de la fédération internationale, en neutralisant justement le fait «national» sur lequel repose la question de la moralité que constitue la tradition culturelle. Dans son texte, Bourne passait évidemment sous silence la nouvelle forme d'impérialisme et de colonialisme qu'exprimaient déjà les États-Unis à ce moment vers l'intérieur comme vers l'extérieur de leurs frontières nationales, mais c'est en fait qu’il s'opposait à la définition du «melting-pot», qui prétendait alors en donner une forme complètement unifiée, en reléguant au second plan la question de la multiplicité culturelle qui en fournissait la structure. Ce débat entre «meltingpot » et «multiculturalisme» a encore cours de nos jours aux États-Unis. 
nationales, voire triturée en son sein par l'opposition catégorique entre «culture nationale » et « civilisation universelle ${ }^{8}$. Le caractère métropolitain de l'Europe s'était ainsi affiché, pendant tout le cours du développement de la modernité, dans des villes-capitales nationales telles Paris ou Londres, devenues des symboles prééminents du cosmopolitisme métropolitain moderne, symboles cependant laissés par la suite derrière de nouveaux développements internationaux où l'espace-temps des villes-métropoles a plutôt essaimé du côté des symboles que sont devenus New York ou Chicago, Berlin ou Vienne, ou encore, plus récemment, Los Angeles ou Mexico ${ }^{9}$. En se diffusant plus largement, en se disséminant dans le monde, l'expérience typiquement moderne du cosmopolitisme a semble-t-il perdu de sa concentration et de sa consistance nationale, gagnant apparemment ainsi en « extension» ce qu'elle perdait en «intention».

La définition contemporaine du cosmopolitisme a donc de toute évidence dépassé les limites que lui imputait la pensée politique moderne, mais à telle enseigne qu'elle apparaît cependant aujourd'hui fractionnée dans des aires d'expériences relativement distinctes et encore seulement partiellement formées; Steven Vertovec et Robin Cohen ont ainsi proposé récemment d'étudier cette redéfinition du cosmopolitisme selon six perspectives, soit: comme condition socioculturelle; comme philosophie ou vision du monde; comme projet politique des institutions transnationales; comme projet politique de sujets multiples; comme attitude ou disposition; et enfin comme pratique ou compétence (Vertovec et Cohen, 2002, p. 11-14). Comme on le verra dans la prochaine section, et en dépit de la difficulté d'unifier ces divers niveaux d'expérience au sein d'une redéfinition synthétique du cosmopolitisme, ce découpage permet au moins d'appréhender sur le plan analytique des pratiques concrètes qui se déploient en conformité avec le développement des relations internationales et transnationales, que l'on retrouve dans les domaines des ententes commerciales internationales et continentales, dans le déploiement transnational de l'industrie et des marchés (du travail, de la consommation et des ressources naturelles), de même que dans les phénomènes mondiaux d'immigration, de formation de diasporas, ainsi que dans le développement plus général d'une vision du monde appelant finalement à redéfinir les termes mêmes d'une compréhension de ce qui a succédé à la modernité proprement dite. Ici, Ulrich Beck a par exemple insisté récemment sur le développement du cosmopolitisme comme étant désormais le seul horizon valable du développement d'ensemble de la société contemporaine, en mettant en relief les pôles positifs et négatifs de ses manifestations actuelles, et en rendant ainsi la projection utopique kantienne à ses conditions

8. En effet, il n'y a jamais eu d'unanimité parfaite quant à la signification du cosmopolitisme dans le développement de la modernité, comme en témoigne notamment, et de manière très éloquente, le débat entre «culture » et «civilisation » qui va opposer, dès la fin du Xviñ siècle, des penseurs comme Herder et Kant. Un tel débat va d'ailleurs rejaillir dans le contexte contemporain à propos de la signification du cosmopolitisme des villes-métropoles, comme on le verra plus bas dans la manière dont est débattue la signification de la ville de Berlin opposant les vues respectives de Werner Sombart et de Georg Simmel.

9. Sur l'évolution du concept de ville-métropole, voir notamment Sutcliffe (1984) ainsi que Scott et Soja (1998), et García Canclini (1998). 
effectives de lutte politique sous l'emprise du mouvement général de la mondialisation ${ }^{10}$. À terme, on peut même dire que ce qui est en jeu est la redéfinition d'un ordre cosmopolitique qui soit également délesté de son allégeance stricte aux principes de la modernité européenne bourgeoise, voire de sa référence obligée à la seule tradition occidentale.

En effet, en récupérant par exemple la signification des éléments historiques et anthropologiques « extérieurs » à la modernité européenne et à la tradition culturelle occidentale, et en les inscrivant dans le registre de préoccupations proprement politiques, certaines réflexions sur le cosmopolitisme contemporain cherchent à élargir le sens de ses applications ${ }^{11}$. Mais de cette façon, la lecture d'une « histoire universelle» qui se révélerait au travers de cet ordre cosmopolitique semble devenir encore plus problématique, ou à tout le moins, elle renvoie à un schéma d'évolution historique dont il reste encore à approfondir les racines anthropologiques (Jaspers, 1954); le «sujet de l'histoire» qui s'y dévoilerait demeure ainsi toujours relativement énigmatique, alors que c'est pourtant lui qu'on retrouve au sein de l'existence des métropoles contemporaines. Mais c'est peut-être alors justement ce trajet d'évolution historique du développement des villes-métropoles qui en donne la trame la plus explicite, et qui est à même de nous renseigner davantage sur la contribution de ces dernières à la configuration du cosmopolitisme contemporain ${ }^{12}$. Les villes-métropoles étant les dépositaires privilégiés de ces développements liés au phénomène de la mondialisation, ce sont elles en effet qui paraissent être à l'avant-plan de la représentation du cosmopolitisme contemporain.

Car cette empreinte du cosmopolitisme contemporain a joué de manière véritablement déterminante dans le développement des villes et des métropoles, en modelant justement leurs visages et leurs formes, en les défigurant parfois autant qu'elle les transformait au gré des événements historiques internationaux - la ville-métropole de Berlin apparaissant

10. Il écrit à ce sujet : «L'enjeu du débat sur la mondialisation n'est pas la signification de l'État national et de sa souveraineté (...), mais l'instauration d'une nouvelle perspective cosmopolitique permettant d'envisager le champ du pouvoir dans son ensemble, et d'appréhender les nouveaux acteurs, réseaux d'acteurs, chances d'accéder au pouvoir, stratégies et formes d'organisation d'une politique qui ignore désormais les frontières : la critique cosmopolitique de la politique et de la politologie centrées sur l'État national et cimentées par celui-ci (...) est fondamentale tant sur le plan empirique que politique. L'argument est doublement piquant : l'épreuve de force est ouverte sur la scène politique mondiale, et elle exige l'adoption d'une orientation cosmopolitique de la théorie et de la pratique sociologique. Celle-ci permettra de mettre en évidence l'ambivalence que comporte cette nature ouverte des fondements comme objectifs et des alternatives de la politique mondiale, et de donner une forme politique à cette ambivalence. » (Beck, 2003, p. 84-85)

11. Je pense notamment ici aux essais regroupés dans Breckenridge et alii. (2002).

12. Je rejoins ici, au moins en partie, un argument développé par Edward W. Soja (2000), qui voit dans la dynamique du développement des villes, mais particulièrement dans leur mouvement d'unification, depuis la plus haute antiquité, le symbole d'une histoire de la civilisation. Une des premières figures à avoir insisté sur ces rapports dans le contexte contemporain est bien sûr Jane Jacobs (1969). On entre en fait ici dans un débat qui est déjà très ancien, à savoir ce qui nous permettrait de trancher la question de la contribution du développement de la ville (et surtout des formes de ce développement) à la civilisation. Ici, autant Lewis Mumford (1940) qu'Oswald Spengler (1948) deviennent des références obligées - et pour ainsi dire complémentaires, puisqu'ils s'accordent tous deux à associer le développement des métropoles contemporaines à une profonde décadence civilisationnelle. 
alors évidemment comme un témoignage particulièrement tragique de toute cette évolution historique $\mathrm{du} \mathrm{xx}^{\mathrm{e}}$ siècle, et même peut-être comme le théâtre métropolitain où se sont joués justement certains de ses épisodes les plus cruciaux. Ce n'est pas une petite ironie en effet que Berlin ait été le lieu de passage le plus intense des deux guerres mondiales, des destructions massives qui les ont accompagnées, de la période nazie entre celles-ci, de la guerre froide hautement symbolisée par la division de la ville par le mur, et enfin de la Pax americana, pour se retrouver au début du $\mathrm{xxI}^{\mathrm{e}}$ siècle en position de devenir pratiquement la capitale de l'Europe. En tant que ville-métropole, Berlin offre ainsi un condensé de toute l'histoire continentale européenne des $\mathrm{xIx}^{\mathrm{e}}$ et $\mathrm{xx}^{\mathrm{e}}$ siècles, inscrite dans les formes mêmes de sa définition spatio-temporelle fracturée par ces divers déchirements, qui sont à leur tour recomposés dans une facture contemporaine. Et le cosmopolitisme qu'elle a développé en tant que ville-métropole tient notamment à cette expérience contemporaine qui a définitivement laissé derrière elle les limites proprement modernes du cosmopolitisme, telles qu'elles avaient par exemple été exprimées par Kant, pour s'ouvrir à des réalités nouvelles qui sont à l'image du monde actuel, et qui forment ainsi le véritable spectacle du monde où se profilent les nouvelles formes de cosmopolitisme.

Ce n'est donc pas un hasard si Georg Simmel avait pu voir déjà dans le Berlin du début $\mathrm{du} \mathrm{xx}^{\mathrm{e}}$ siècle la représentation par excellence du cosmopolitisme; cette représentation tenait alors autant au caractère inouï du développement métropolitain qu'il avait sous les yeux, qu'à la signification politique universelle qu'il semblait alors promettre, dans la mesure de sa capacité à réfléchir les développements historiques du monde contemporain. C'est d'ailleurs la raison précise pour laquelle une telle vision pouvait être contestée à l'époque d'un point de vue plus «national », de la part par exemple de Werner Sombart, qui voyait de son côté en Berlin le lieu de l'aliénation et de la dépossession de l'identité culturelle allemande. Comme l'a rappelé l'analyse rétrospective de ce débat autour de la signification de la ville de Berlin de David Frisby, la dénonciation du caractère « universel » (ou même " américain») de la métropole allemande par Sombart se faisait alors au profit de l'affirmation du caractère véritablement «allemand» de... Vienne (Frisby, 2001, p. 159179). Les événements historiques qui allaient succéder à ces disputes de représentation n'allaient pas aider à éclaircir la situation, redoublant même la complexité de l'analyse de Berlin en tant que ville-métropole, dont la signification symbolique demeure peut-on dire jusqu'à aujourd'hui toujours problématique. Et cela n'est pas dû au fait de la distorsion introduite par exemple par la désignation de Bruxelles comme capitale politique de l'Europe, en tant que ville-hôtesse de la Commission européenne, ou Strasbourg, en tant que lieu parlementaire de l'Union européenne, au détriment de la place réelle qu'occupent toujours d'autres villes européennes qui semblent posséder davantage de rayonnement. En effet, si un des aspects centraux de Berlin apparaît maintenant être ce «spectacle de la destruction », qui transparaît autant dans les phases successives de la fulgurante reconstruction de l'après-Deuxième Guerre et de l'après-réunification, que dans un imaginaire artistique de la ruine et des cimetières inexorablement lié à la ville, il devient difficile de voir comment on peut envisager qu'elle soit simultanément le symbole d'un avenir 
universel $^{13}$. Et pourtant, c'est bien ce sens qui est envisagé, non seulement dans la foulée du mouvement de la mondialisation qui reconfigure par exemple la Potsdamer Platz en arène publicitaire des corporations transnationales, mais également dans la reconstruction du Reichstag à l'image d'une " démocratie nationale transparente», de même que dans les efforts gigantesques consentis par les différents ordres de gouvernement à l'égard du développement culturel de la métropole berlinoise - tout autant par ailleurs que dans les flux migratoires, le développement de l'underground artistique et les recompositions du tissu humain de la ville ${ }^{14}$. L'ironie de la situation se mesure en effet à une transparence présumée de la politique nationale, au moment précis où celle-ci perd de son emprise au profit du mouvement de la mondialisation, à l'avènement des représentations publiques des corporations transnationales agissant toujours au nom d'intérêts particuliers seulement, et finalement, à la cacophonie des recherches médiatiques et artistiques tiraillées dans leurs allégeances diverses aux motivations qui les suscitent, à travers les transformations inhérentes à l'espace public et à la définition du caractère culturel de la ville.

Ce qui se produit en réalité au travers ces télescopages historiques et ces transformations épistémiques, c'est bien une situation «traumatique», mais celle-ci peut aisément être relayée par une capacité technique experte de jouer sur les représentations afin d'en tirer une perception unifiée et, pour ainsi dire non problématique, voire proprement spectaculaire $^{15}$ : Berlin présenterait donc de facto la définition du cosmopolitisme contemporain. Or, c'est justement dans l'inversion de cette non-problématicité de l'espace-temps métropolitain, ainsi que dans la révélation de son caractère dialectique, voire par moments aporétique, que logent les possibilités de redéfinition du cosmopolitisme; si celui-ci apparaît en effet dans certaines formes culturelles contemporaines, il s'y exprime d'une manière nouvelle, mais il demeure toujours cependant problématique, voire éminemment contradictoire, dans ses représentations. C'est que le cosmopolitisme est rendu, aujourd'hui, à sa définition réellement dialectique dans la discussion politique qui se développe à son

13. Régine Robin écrit à ce sujet: "Les chantiers aux centaines de grues seraient-ils aussi des dépotoirs, des décharges, des lieux de démémoire où les nouvelles constructions sorties de terre se mélangeraient avec les "poubelles de l'histoire" qui, à Berlin plus qu'ailleurs, sont pleines à craquer ? Si on voulait bien trier ces ordures (une obsession écologique bien berlinoise puisque chacun a au moins quatre boîtes à ordures à sa disposition), on trouverait aussi les déchets, décombres et rebuts d'une amnésie programmée, celle d'un mauvais rêve qu'on n'arrive plus à transformer en cauchemar : la République démocratique allemande. (Robin, 2001, p. 116-167) Sur la question de l'esthétique de la ruine et du cimetière, liée à la perte d'identité et à la faillite morale, voir Dion (1999).

14. Voir entre autres à ce sujet Grésillon (1998). La situation de Berlin est sans doute exceptionnelle en ce qui a trait à l'investissement des ressources financières sur le plan culturel - et cela se comprend autant d'un point de vue historique que plus étroitement idéologique -, mais cela traduit en fait surtout une tendance contemporaine à insister sur la mise en valeur symbolique de lieux spécifiques. Voir à cet égard Dietger Pforte (1991), ainsi que Grezner (2001; 2002).

15. Mark Seltzer écrit à ce sujet: " Hence what I have been tracing, by way of the situation of Berlin, are in effect two versions of the urban after-image, which are also, and crucially, two versions of the condition of publicness today : the traumatic and the forensic. (...) The trauma apriori should by now be clear enough. (...) What has been described as the emergence of a "new victim" order, an order that proceeds under the sign of an ecumenical pathos, amounts to the positioning of the sociality of the wound: collectivity in commiseration. (...) Beyond that, the forensic way of seeing pathologizes, or criminalizes, public space.» (Seltzer, 2003, p. 71) 
endroit; les formes expressives et contradictoires qui se déploient dans les villes-métropoles témoignent éloquemment de cela.

Les villes-métropoles contemporaines, comme Berlin, permettent en fait le développement de formes culturelles hybrides qui, dans les tensions mêmes qui les constituent, s'ouvrent concrètement au questionnement du politique à travers des pratiques quotidiennes, lorsque celles-ci révèlent des significations internationales; à Berlin par exemple, la communauté turque apparaît ainsi, dans le développement de ses pratiques médiatiques, témoigner des possibilités de transformation et d' «incorporation» des traits à la fois étrangers et familiers à partir desquels s'apprivoise, se domestique et se cultive la vie sociale et politique des villes-métropoles dans une perspective «transnationale», en transformant toutefois évidemment la signification moderne du cosmopolitisme, sans en donner pour autant la signification achevée (Caglar, 2002). Berlin est également devenu, particulièrement depuis l'ouverture du mur et du questionnement politique qu'il a suscité, un point de chute pour l'underground artistique européen et mondial - et les enjeux idéologiques présents à cet égard n'y sont certainement pas étrangers. De même, la ville s'est affichée comme une référence internationale de certains mouvements culturels, tels ceux incarnés par la scène musicale techno, la «gay pride» ou encore le mouvement écologique. Et ce sont là des exemples de réflexions internationales situées dans le milieu de la ville-métropole que l'on peut retrouver autant à Londres qu'à Paris ou Marseille, autant à New York qu'à Montréal, autant à Shanghaï qu'à Hong Kong, selon des modalités variables (Abbas, 2002). De cette façon, rejaillissent même parfois les enjeux d'une révision des rapports de domination issus de situations coloniales, qui situaient également les termes du cosmopolitisme moderne, mais en dehors des conflits strictement guerriers qui pouvaient (ou qui pourraient) tout autant en déterminer l'issue, puisque les villes-métropoles incarnent désormais en elles-mêmes toutes sortes d'oppositions entre dominants et dominés, tout en les faisant cohabiter. Les villes-métropoles deviennent ainsi le miroir réfléchissant des tensions du monde dans le plus près de leurs activités quotidiennes, ainsi que le lieu concret d'élaboration des nouveaux rapports politiques structurant la vision du monde que l'on peut avoir aujourd'hui, puisqu'elles sont le milieu de leurs affrontements directs, c'est-à-dire donc l'espace-temps d'affrontement et de médiation symbolique par excellence où ces oppositions se manifestent dans leur recherche de cohabitation.

L'idée que je voudrais avancer ici est donc précisément que tous les enjeux liés au développement du cosmopolitisme contemporain se sont trouvés en quelque sorte condensés et sédimentés dans le développement des villes-métropoles, à cause du croisement en leur sein des grandes forces historiques des deux derniers siècles, ainsi que de l'évolution de la politique internationale, mais d'une manière qui fait ressortir autant leur proximité que leur distance par rapport à la définition moderne et à ses dépassements possibles qui viennent d'être résumés ici à grands traits et très rapidement. En effet, pendant que se déployaient ces grands enjeux historiques d'orientation du monde à travers les relations politiques internationales contemporaines, se tramait l'existence des villes-métropoles au sein des deux phénomènes majeurs qui ont rejoint l'évolution de la forme urbaine depuis les deux derniers siècles: l'industrialisation et l'urbanisation. Ces deux phénomènes, couplés aux aléas des guerres, des relations internationales, de la formation des organisations 
internationales, et même de la Pax americana, ont structuré l'espace-temps contemporain des villes-métropoles, en permettant l'expression d'une expérience humaine déployée dans tous les horizons de cette «insociable sociabilité» dont parlait Kant, mais aussi selon une transformation du sens de cette expérience appelant la définition de termes inédits pour l'appréhension des formes nouvelles du cosmopolitisme. On voit peut-être ici également comment, lorsque les phénomènes d'urbanisation et d'industrialisation sont pensés indépendamment l'un de l'autre, ou dans une totale soumission de la première à la seconde comme Marx l'a fait à sa manière, en même temps que tout le courant de l'urbanisme, mais de manière antithétique ${ }^{16}$-, on manque d'appréhender les conditions politiques internationales qui en structuraient a priori les définitions - comme le fait par exemple David Harvey (1992) dans le contexte postindustriel contemporain. Car en dépit de sa distance avec la ville au sein de l'État-nation contemporain, la définition de la citoyenneté nationale et internationale donnant sa signification au cosmopolitisme s'est en fait pendant ce temps rapprochée au plus près de l'expérience concrète, pour ne pas dire de l'expérience quotidienne, des villes, et particulièrement des métropoles, dans le développement précis de leurs formes.

Je passerai maintenant à l'examen succinct de cette question, qui tient inévitablement à la possibilité de recomposer une vision du caractère spécifique du cosmopolitisme métropolitain, mais auparavant, je donne un bref exemple de la manière par laquelle le cosmopolitisme ne peut aujourd'hui se contenter d'une vision simplement « réalisée » de ses fins. Dans un texte écrit au milieu des années 1970, Peter Berger livrait une analyse de la ville de New York qui confondait ses caractéristiques sociologiques et théologiques; pour lui, s'il existait un exemple dans le monde correspondant à la vision d'un cosmopolitisme réalisé ( «... the city par excellence, the prototypical cosmopolis of our age»), on le trouvait dans les formes de cette vie urbaine pouvant faire penser que la «cité céleste», celle entrevue notamment par les traditions religieuses judéo-chrétiennes, touchait réellement, à New York même, ses éléments de concrétisation graduelle, faisant de cette dernière le nouvel emblème de la cité édénique ${ }^{17}$; les événements de septembre 2001 nous ont rappelé à cet effet, et de manière assez brutale, que tout symbole cosmopolitique dans le monde contemporain ne pouvait se contenter d'une telle vision positive et idyllique, et devait justement en réfléchir plutôt toutes les contradictions.

16. Il est frappant en effet de voir comment l'urbanisme, en tant que courant de planification urbaine à tendance «scientiste» se développant à partir de la fin du $\mathrm{XIX}^{\mathrm{e}}$ siècle, a constamment écarté de ses analyses la dimension politique de la ville. À l'opposé, Marx mettait en avant-scène de ses analyses les rapports contradictoires de l'économie politique, sans relever de manière significative les dispositions urbaines de leur réalisation. Certaines tentatives, comme celle de Henri Lefebvre, ont insisté sur la possibilité de médiation de ces deux tendances antithétiques, mais en ne renouvelant pas de manière systématique l'assise épistémologique dont procédaient, chacun de son côté, l'urbanisme et le marxisme.

17. Voir Berger (1977). Dans un esprit moins « illuminé», Thomas Bender a récemment insisté sur le projet cosmopolitique new-yorkais, toujours à reprendre en fonction de ses incomplétudes passées - voir en particulier le chapitre «Cities and Citizenship» (Bender, 2002, p. 199-217). 


\section{LES ESPACES-TEMPS FRACTION NÉS DES MÉTROPOLES CONTEM PORAINES ET LES NOUVELLES FORMES DU COSMOPOLITISME}

Réfléchissant sur les propositions de Georg Simmel en rapport à la signification du caractère métropolitain de Berlin, Richard Sennett a proposé récemment l'idée que les caractéristiques principales du cosmopolitisme contemporain logeaient désormais du côté de l'« indifférence» et de la «flexibilité » liées au développement du capitalisme des corporations transnationales; à ses yeux, là où Simmel pouvait relever les dimensions d'altérité et de rationalité comme composantes essentielles de la ville-métropole du début du $\mathrm{xx}^{\mathrm{e}}$ siècle, en tant que caractéristiques liées au développement économique capitaliste, nous devrions aujourd'hui compter sur leur substitution par des rapports d'indifférence dans la localisation des activités économiques et de flexibilité dans leur organisation comme facteurs de «détachement» du cosmopolitisme vis-à-vis de la ville (Sennett, 2002 ${ }^{18}$ ). Cette idée, couplée à celle d'une seule possibilité d' «identification temporaire » à la ville-métropole, renvoie au fait, central pour l'analyse qu'en fait également par exemple David Harvey, que la rationalité capitaliste postfordiste annihilerait toute possibilité de déploiement d'une « vision du monde» autre que celle immédiatement compressée, dans les termes abstraits du profit présentés aujourd'hui, dans sa forme d'accumulation «flexible » - et «spectacularisée » dans les formes de la culture métropolitaine ${ }^{19}$. Nous serions ainsi en présence d'un cosmopolitisme totalement désincarné et lié seulement aux activités des dirigeants, voire de la simple logique, des corporations transnationales, d'une part, ainsi que, d'autre part, de villes livrées avant tout et principalement aux effets «spectaculaires» du capitalisme - effets qui sont eux-mêmes relevés dans l'analyse, c'est-à-dire portés à la représentation comme étant les caractéristiques principales du spectacle des villes-métropoles contemporaines. Du point de vue de la perception des éléments qui entrent dans la composition du cosmopolitisme contemporain, les choses m'apparaissent cependant plus complexes ; la dialectique inhérente au développement des formes de la ville-métropole contemporaine ne se réfléchit pas simplement en une "annihilation de l'espace par le temps », selon la formule synthétique de David Harvey, ce qui rendrait impossible la composition d'une représentation politique concrète, mais bien au contraire par ce qu'on pourrait appeler une « dia-topie » et une « dia-chronie», c'est-à-dire une séparation de l'espace et une séparation du temps, processus de divisions et d'oppositions qui font proliférer des spa-

18. Dans son argumentation, Sennett prend appui notamment sur le fait que les dirigeants des grandes corporations ne s'impliquent plus activement dans la vie civique et politique, au sein des conseils municipaux ou des conseils d'administration et de gestion des services publics, comme ils le faisaient auparavant, sans relever le fait que ce sont désormais nécessairement des citoyens ordinaires qui le font ; peut-on alors vraiment parler en cela d'une baisse de l'implication politique de la part des dirigeants économiques, ou ne doit-on pas y voir au contraire une démocratisation de la vie politique dans son contexte urbain?

19. "The image, the appearance, the spectacle can all be experienced with an intensity (joy or terror) made possible only by their appreciation as pure and unrelated presents in time. (...) The immediacy of events, the sensationalism of the spectacle (political, scientific, military, as well as those of entertainment), becomes the stuff of which consciousness if forged. » (Harvey, 1992, p. 54) 
tialités et des temporalités diverses et souvent contradictoires, qui font même se côtoyer ces dernières dans une rencontre (parfois brutale) de leurs significations respectives à travers les formes d'existence et d'expérience urbaines qu'elles développent. Ce sont les affrontements de ces significations qui deviennent, et justement à cause de cela, les éléments de base d'une représentation véritablement cosmopolitique de la ville-métropole.

Ainsi, dans les villes-métropoles contemporaines, au sein même de la foule anonyme, les dirigeants des entreprises transnationales croisent les sans-abri, les touristes du monde fréquentent les «spécialités locales», les immigrants travaillent dans l'industrie hôtelière au service des voyageurs de toutes sortes, les représentations médiatiques livrent une guerre de tranchée aux initiatives artistiques, les « cuisines ethniques » proposent aux « habitants locaux» des saveurs du monde, bref, c'est le mélange le plus éclaté qui définit le spectacle du monde; et c'est donc lui qui devrait conduire nos conceptions du cosmopolitisme ${ }^{20}$. Le cosmopolitisme apparait toutefois dans ce contexte non pas comme une capacité de rendre compte de ces fractionnements spatiotemporels, mais bien plutôt d'une capacité de les médier les uns les autres (ou d' «arbitrer» ces différences en leur donnant une possibilité de médiation), en saisissant leurs « collisions » comme étant des indices de la problématique concrète ouverte aujourd'hui à la réflexion ${ }^{21}$. Le spectacle des villes ne se donne pas alors dans la forme simplifiée d'un cosmopolitisme réalisé de manière unilatérale et «spectaculaire» (comme dans sa version économique capitaliste), mais bien, et inversement, dans la forme politique problématique des fractures spatiotemporelles dont les villes-métropoles sont les réceptacles et les emblèmes, en tant que lieux de dévoilement d'un ordre cosmopolitique aujourd'hui encore et toujours anonyme; davantage, c'est cet anonymat même, cette " absence de nom», qui permet la localisation du caractère universel que possède le cosmopolitisme, dès lors qu'il apparaît dans une représentation faisant état de sa propre problématicité au sein de la vie métropolitaine. Ainsi, toute ville-métropole dévoile un aspect du cosmopolitisme contemporain dans ce spectacle problématique pris en charge par sa représentation - et c'est à cette occasion que le cosmopolitisme sort de

20. "Cosmopolitanism in the Midle East, in the old-fashioned sense of communally deracinated and culturally promiscuous groups and milieux, continues to exist in particular corners of urban space. These, however, are submerged by the two major forces of the metropolis: the recent urbanized masses and their transformation of the city and its politics; and forces of international capital, of business and tourism and their towering buildings, hotels and offices, their media and the consumption goods and images for which they cater. » (Zubaida, 2002, p. 41)

21. Je me réfere ici à la thématisation des « collisions éthiques » qui se produisent justement au moment où, dans l'ordre culturel contemporain, les discours sur la ville établissent en apparence un espace territorial unifié, et cela pratiquement mondialement, alors même que les actions concrètes et les activités urbaines en général font plutôt état d'un intense fractionnement localisé dans la signification donnée à l'existence métropolitaine. Voir à ce sujet Blum (2003, p. 48-49). Dans un esprit similaire, Ackbar Abbas écrit : « ... the cosmopolitan today will include not only the privileged transnational, at home in different places and cultures, as an Olympian arbiter of value. Such a figure, it could be argued, has too many imperialistic associations. The cosmopolitan today will have to include at least some of the less privileged men and women placed or displaced in the transnational space of the city and who are trying to make sense of its spatial and temporal contradictions : the cosmopolitan not as a universalist arbiter of value, but as an arbitrageur/arbitrageuse.» (Abbas, 2002, p. 226) 
son anonymat pour devenir emblématique d'une situation liée à une ville-métropole en particulier, faisant de celle-ci une de ses représentantes. Mais la ville-métropole ne peut jamais, dans ce contexte et pour cette raison même, que donner une mesure fragmentaire du cosmopolitisme. C'est la raison pour laquelle les espaces-temps de représentation de la ville-métropole se donnent aujourd'hui dans les termes où leurs contradictions importent plus au fond que leurs définitions unifiées, puisque ces espaces-temps fracturés donnent ainsi une occasion de saisir les aspects manquants d'une médiation symbolique projective de ce qu'est véritablement le cosmopolitisme dans sa capacité de réfléchir politiquement les conflits, c'est-à-dire de les révéler tels qu'ils manifestent, d'abord esthétiquement, dans les formes de leurs expressions, une exigence de médiation éthique.

Il faut en effet rappeler d'abord ici que les villes contemporaines se sont distinguées de leur définition moderne en accord avec le développement d'une société de masse qui s'est graduellement imposée en laissant derrière elle certains des principes fondamentaux de la société moderne bourgeoise ${ }^{22}$. C'est par un curieux paradoxe en effet que la société bourgeoise, en donnant naissance à l'État-nation, a fait en sorte de permettre que les villes, qui avaient assuré le développement initial des bourgeoisies modernes depuis la fin du Moyen-Âge, à la Renaissance et pendant tout le cours de la modernité, deviennent désormais plutôt le lieu d'apparition d'un tout autre développement lié, lui, aux masses d'individus indifférenciés qui se trouvent à envahir l'horizon social à travers une transformation profonde de leur existence et de l'expérience humaine. La sociologie, au tournant des $\mathrm{XIX}^{\mathrm{e}}$ et $\mathrm{XX}^{\mathrm{e}}$ siècles, a enregistré ces transformations, au-delà de ses appréhensions initiales suscitant des antagonismes catégoriques de différentes façons; des études sur la foule de Gustave Le Bon et de Gabriel Tarde, en passant par les travaux de Robert E. Park sur la ville, et jusqu'aux évaluations d'un nouveau type social chez le David Riesman de The Lonely Crowd, c'est en effet tout le trajet d'émergence de la société de masse, telle qu'on la trouve en particulier dans les villes-métropoles contemporaines et dans la consolidation de ses nouvelles catégories de référence, qui s'écrit. Le lien entre les villes-métropoles et les nouvelles formes de cosmopolitisme s'établit donc de manière obligée par ce passage à travers le phénomène de la masse, ou plus exactement, de la société de masse, puisque c'est elle qui fournit l'anonymat universel à travers lequel peut se manifester le caractère représentatif de la personne (catégorie au fondement de l'ordre politique universel). La villemétropole acquiert ainsi une sorte d'indépendance relative vis-à-vis de l'ordre politique général dans la manière dont elle permet ces manifestations, en même temps que l'ordre politique universel développe une dépendance directe vis-à-vis des villes-métropoles dans la concrétisation de sa définition complète. Ici, comme dans l’ordre politique en général, la figure du bourgeois ainsi que ses modes de représentation ont été remplacés par la figure de la personne et ses modes propres de présentation. Et cela ne signifie pas simplement la «chute de l'homme public», dans la désintégration de la sphère publique

22. Voir à cet effet Freitag (1971, p. 25-57), qui donne une version cependant unilatéralement négative de ce mouvement. 
bourgeoise, comme le laissaient entendre Richard Sennett (1974) ou Jürgen Habermas (1993), mais bien plutôt l'ascension de la «personne anonyme» comme fondement universel de l'agir social dans tous les moments et les lieux de sa manifestation, ainsi que la recomposition d'un espace de représentation politique succédant à celui de la société moderne. Et les villes-métropoles sont devenues le lieu de manifestation par excellence de cette expérience politique universelle dans sa multiplicité. Dans son texte «Métropoles et mentalité », Georg Simmel avait déjà donné une expression singulièrement frappante de cet affrontement entre le monde bourgeois et la société de masse, qui résulte en fait en une définition de la nouvelle problématique du cosmopolitisme dans son lien autant à l'histoire universelle qu'à l'expérience individuelle, ces deux termes étant alors médiatisés justement par la métropole comme espace de liberté. Simmel écrivait à ce sujet:

Si l'on s'interroge sur la position historique de ces deux formes d'individualisme qui se nourrissent des rapports quantitatifs de la grande ville: l'indépendance individuelle et la formation de l'originalité personnelle, alors la grande ville gagne une valeur tout à fait nouvelle dans l'histoire mondiale des mentalités. Le XviII ${ }^{\mathrm{e}}$ siècle trouvait l'individu retenu par des liens d'ordre politique et agraire, corporatif et religieux, qui lui faisaient violence et qui avaient perdu tout sens. Ces oppressions imposaient à l'homme, pour ainsi dire, une forme «non naturelle » et des inégalités depuis longtemps injustifiées. C'est dans cette situation qu'est né l'appel à la liberté et à l'égalité - la foi dans la pleine liberté de mouvement de l'individu dans les rapports sociaux et intellectuels, apparue en même temps dans le peuple et chez les nobles, comme si la nature l'avait déposée en chacun et que la société et l'histoire l'avaient déformée. En plus de cet idéal du libéralisme, grâce à Goethe et aux romantiques d'une part, un autre idéal se forme au XIX ${ }^{e}$ siècle: libérés des liens historiques, les individus veulent aussi à présent se différencier les uns des autres. En chaque individu, ce n'est plus l' «universalité de l'homme», mais précisément l'unicité qualitative et le caractère irremplaçable qui constituent à présent les supports de sa valeur. C'est dans le conflit et les entrelacs changeants de ces deux modes de détermination du rôle du sujet dans la collectivité que se développe l'histoire externe et interne de notre temps. (Simmel, 1984, p. 76)

Cette exigence de « différence individuelle » à l'égard de l'universalité anonyme de la masse définit la liberté citoyenne contemporaine, dans la mesure d'un affrontement direct qui rejoint surtout l'individu métropolitain; Simmel fait donc apparaitre ici le rôle essentiel des villes-métropoles, où se concentre la tension entre «l'atrophie de la culture individuelle par l'hypertrophie de la culture objective», soient les forces de l'économie, de la division du travail et celles de la technique, qui affrontent celles de la volonté subjective de la personne dans ses capacités expressives, normatives et cognitives. Et ce rapport de forces se joue justement sur la capacité d'exacerbation des termes contraires, où le nivellement anonyme de l'existence métropolitaine générale dans les phénomènes de masse engendre les plus grandes capacités d'expression de la subjectivité individuelle, qui se déploie ainsi jusqu'à l'excentricité. À l'inverse de la tension précédente, ainsi, qui voyait les grandes lignes de l'évolution sociohistorique rencontrer le plus profond de l'expérience subjective individuelle au sein de la métropole, celle-ci devient justement à ce moment le révélateur d'une condition générale du cosmopolitisme, par le biais entre autres de l'expression de 
l'excentricité subjective individuelle liée aux possibilités de la ville-métropole. C'est ainsi que la «nature», non plus «physique» ou «théologique», comme chez Kant, mais bien désormais entièrement sociale de l'expérience «massive» de la métropole rejoint la «seconde nature» (soit la définition symbolique) de l'individualité sociale personnelle, pour nouer le faisceau des expériences diverses qui font apparaître les nouvelles formes du cosmopolitisme contemporain.

Ces formes sont diverses, mais elles ressortissent toutes par ailleurs du même rapport qui se noue ici entre l'évolution sociohistorique du monde contemporain d'un côté, et de l'individu de l'autre, rapport concentré dans l'existence des villes-métropoles qui lui fournissent la «scène » de son développement, soit la base de représentation à partir de laquelle sont joués les conflits dramatiques de l'existence humaine; cette dialectique réfléchit également en effet toujours le conflit de l'«insociable sociabilité », et le réfléchit dans des conditions d'expériences données, opérant la traduction symbolique des forces en présence dans le cours de l'évolution du monde contemporain désormais mis en face de lui-même seulement, et non plus d'une simple projection utopique ou théologique, pour la mise en forme de sa propre réflexivité. C'est ici que l'on peut revenir sur les différentes perspectives ouvertes sur le cosmopolitisme contemporain de Vertovec et Cohen énoncées précédemment, mais en insistant sur le contenu qu'elles révèlent dans le contexte des villes-métropoles, puisqu'on appréhende par là des aires de pratiques qui renvoient à une anthropologie de la vie urbaine comme point d'appui de la représentation du monde ${ }^{23}$. C'est ici que se déploient les diverses conditions socioculturelles des populations, en révélant les virtualités des divers projets politiques, et que s'actualisent les dispositions et attitudes cosmopolitiques dans les termes des pratiques et des compétences toujours fragmentaires, certes, mais pourtant ouvertes sur un horizon universel.

J'ai mentionné plus haut comment des événements historiques tels que les guerres avaient transformé, parfois jusqu'à les défigurer, les métropoles contemporaines; mais les guerres militaires sont à cet égard l'équivalent des guerres sociales et culturelles internes qui ont transformé la sensibilité et les mœurs des habitants des villes, et des métropoles en particulier, en leur donnant de nouvelles figures également. C’est ainsi, par exemple, que le phénomène de la guerre a trouvé son équivalent symbolique dans les «guerres esthétiques» menées par les diverses avant-gardes artistiques contre la culture bourgeoise, et ce depuis le milieu du XIX ${ }^{\mathrm{e}}$ siècle. Ces guerres artistiques, dont on perçoit les traces visibles et audibles dans la peinture, la musique, la littérature, l'architecture, constituent

23. Comme l'écrivent Michel de Certeau et Luce Giard : «Les artistes quotidiens des manières de parler, de se vêtir, d'habiter sont des revenants dans l'art contemporain patenté. Il serait grand temps qu'un urbanisme encore en quête d'une esthétique leur reconnaisse la même valeur. Déjà la ville en est la permanente et mobile exposition. Mille façons de s'habiller, de circuler, de décorer, d'imaginer tracent les inventions nées de mémoires inconnues. Fascinant théâtre. Il est composé de gestes innombrables qui utilisent le lexique des produits de consommation pour donner langage à des passés étranges et fragmentaires. "Idiolectes" gestuels, les pratiques des habitants créent, sur le même espace urbain, une multitude de combinaisons possibles entre les lieux anciens (secrets de quelles enfances ou de quelles morts?) et des situations nouvelles. Elles font de la ville une immense mémoire où prolifèrent des poétiques. » (Certeau (de), Giard, Mayol, 1994, p. 201) 
maintenant autant de symboles de la mise en place des nouveaux espaces-temps de l'expérience esthétique, et elles ont fait des artistes les porteurs de certaines des nouvelles formes d'expression du cosmopolitisme, en ramenant l'expérience de l'art à ses conditions d'expérimentation anthropologique au sein de la subjectivité individuelle en quête d'une universalité expressive - symbole d'un véritable cosmopolitisme parce qu'il s'enracine dans les transgressions des frontières socioculturelles traditionnellement reconnues, autant que par l'ouverture de nouveaux territoires symboliques pour la subjectivité. Ici, toutes les recherches et toutes les excentricités commises au nom de l'art par le mouvement général de ce qu'on a appelé, par redoublement de la modernité historique, la «modernité esthétique », ont témoigné de l'essor d'une nouvelle sensibilité au temps et à l'espace, correspondant dans ce sens à ce qu'on peut désigner comme un nouveau rapport au cosmos de l'expérience humaine, c'est-à-dire à son univers propre, énoncé cependant de manière critique dans le contexte où il n'était pas reconnu comme tel (Bell, 1978, p. 33-145). Des commentateurs et analystes récents, tels Fred Jameson (1991) et David Harvey (1992, p. 260-283), se sont d'ailleurs penchés sur ces questions, parfois trop rapidement, en les liant immédiatement à la dépendance économique directe et sans merci que crée le capitalisme. J'irais pour ma part plus loin, ou en tout cas dans une direction autre que celle-là, en appréhendant plutôt les formes de réflexivité produites par la subjectivité individuelle dans ces contextes esthétiques (et éthiques) qui ne sont pas déterminés de manière unilatérale par la dimension économique, mais qui renvoient plutôt à l'ouverture d'une réflexion culturelle et politique à caractère anthropologique rejoignant la structure symbolique de la subjectivité. Ici, les rapports entre l'expérience artistique, que ce soit du côté d'Edgar Allan Poe, de Charles Baudelaire, de Gertrude Stein, de James Joyce, de Robert Musil, et plus récemment de Gerard Richter ou d'Andy Warhol, avec les villes-métropoles, que ce soit Berlin, Paris, Dublin, Vienne ou New York, ou d'autres, n'ont fait que confirmer cette capacité de médiation de l'expérience d'une transformation symbolique intense s'effectuant dans les termes d'une nouvelle forme de cosmopolitisme, où les enjeux de la signification de la nature et de la divinité hérités de la philosophie moderne, tels que saisis par exemple dans leur formulation kantienne, ont été réexaminés à l'aune d'une nouvelle expérience historique et culturelle de l'existence sociale concentrée dans les métropoles comme siège de la nouvelle subjectivitée $e^{24}$. Ici, l'étrangeté des formes d'expression artistique, qui a tant déstabilisé la réception des divers publics des avant-gardes de la modernité esthétique, n'a joué en somme qu'un rôle de révélateur à l'égard de ce que devenait l'expérience de la socialité de masse à travers les métropoles: le rapport dialectique d'identification et de différenciation entre le «soi» et les «autres», et à l'«Autre» de l'ordre

24. C'est dans cet esprit que Malcolm Bradbury écrit, au sujet des rapports entre le modernisme artistique et les métropoles où il s'est localisé: «The modern city has appropriated most of the functions and communications of society, most of its population, and the furthest extremities of its technological, commercial, industrial and intellectual experience. The city has become culture, or perhaps the chaos that succeeds it. Itself modernity as social action, it is both the centre of the prevalent social order and the generative frontier of its growth and change.» (Bradbury, 1983, p. 97) 
symbolique, sous la gouverne désormais de la citoyenneté universelle, se traduisant ainsi sur le plan esthétique en exacerbant l'expression des termes des rapports réciproques de l'individu à la masse, soit l'expression des termes politiques de l'identification générale et universelle au sein même du processus de la différenciation personnelle $e^{25}$. En d'autres termes, on pourrait avancer que plus les villes-métropoles s'ouvraient à l'excentricité des expériences esthétiques dans le déploiement de l'étrangeté liée à leurs formes d'expression, plus elles rendaient propice la définition de formes de cosmopolitisme liées à la figure générale de l'étranger dont les expressions artistiques se faisaient elles-mêmes les lieux de définition ${ }^{26}$.

Bien sûr donc l' «étrangeté » de l'expérience artistique désigne alors une condition humaine universelle, soit celle qui cherche ses repères dans le «nouveau monde » que fait apparaître la société de masse et ses divers avatars (production de masse, consommation de masse, communication de masse, culture de masse, etc.); et cette condition rejoint ainsi justement la grande masse des individus par le biais d'un phénomène lié intrinsèquement au développement de l'urbanisation contemporaine : l'immigration. Que ce soit en effet dans le contexte « intra-national » de l'immigration de la campagne vers la ville, phénomène qui est resté peu étudié dans ses répercussions subjectives, bien qu'il soit le phénomène démographique majeur de la période allant du milieu du $\operatorname{xIx}^{\mathrm{e}}$ au milieu du $\mathrm{xx}^{\mathrm{e}} \operatorname{siècle}^{27}$, ou que ce soit dans le contexte « inter-national», beaucoup mieux connu aujourd'hui à travers la problématique du «multi-culturalisme», des migrations de populations attirées vers des nouveaux centres politiques et économiques, et le phénomène élargi des diasporas et de la problématique des "réfugiés ", ces phénomènes s'imposent dans les métropoles, dès la fin du $\mathrm{XIX}^{\mathrm{e}}$ siècle et jusqu'à aujourd'hui, comme traces extrêmement sensibles d'une

25. J'ai développé l'analyse plus détaillée de cette question dans Côté (2003).

26. Et ici, je rejoins Louis Jacob lorsqu'il écrit, par rapport aux expressions artistiques contemporaines: "Ces expériences axées sur la revitalisation des liens de citoyenneté, des appartenances locales, sur la reconquête de l'autonomie et de la dignité de la personne ont été trop souvent vaincues par l'inertie ou par la pression de logiques adverses, comme celles du spectacle. Pour ne pas les voir condamner à l'autoexaltation et à de brèves poussées médiatiques, et retomber aussitôt dans l'oubli, le travail doit s'accompagner d'un effort parallèle pour lutter contre les causes structurelles, sociales et historiques de l'exclusion. (...) L'idée de cosmopolitisme en art devrait se traduire par un dialogue concret et vivant, par l'expérimentation, par la confrontation de pratiques différentes qui offrent des expériences différentes. L'art serait, comme le politique, un espace ménagé à la problématisation de l'expression, de la communication, de la construction des identités et des volontés.» (Jacob, 2003, p. 45-46)

27. Mais ce phénomène se poursuit toujours aujourd'hui ; la ville de Mexico, par exemple, a vu sa population se multiplier par 15 depuis les années 1940 ; la population y est en effet passée de 1,2 million à 18 millions - voir à cet effet Nestor García Canclini (1998, p. 15-39). Cet exemple peut être multiplié, et il renvoie en fait à un modèle de développement urbain qui situe la ville-métropole comme principal pôle attracteur des populations; ici, la ville-métropole devient l'emblème du mouvement de transformation de l'agriculture et de l'industrie, de même que du commerce, et donc de l'économie capitaliste dans son ensemble, mais elle apparaît autant comme la médiatisation politique de ces diverses transformations dans le développement de son être même. 
transformation de l'existence sociale citadine ${ }^{28}$; toutes les populations qui ont migré vers les villes et les villes-métropoles au $\mathrm{xx}^{\mathrm{e}}$ siècle ont vécu ce passage du connu à l'inconnu, cette excursion, plus ou moins provisoire, plus ou moins permanente, à travers laquelle elles appréhendaient l'étrangeté de leur nouveau milieu de vie, en même temps que ce milieu de vie accueillait en lui l'étrangeté de ces populations mêmes. Le visage des métropoles s'est ainsi (con)formé à l'image de ces foules cosmopolites, c'est-à-dire des masses d'individus dont l'apparence globalement anonyme se comprenait essentiellement à travers la diversité de leurs provenances sociales et culturelles (pour ne pas dire personnelles et historiques), c'est-à-dire à travers les horizons multiples de leurs histoires individuelles, réfléchissant essentiellement les grands mouvements nationaux et internationaux de population conditionnant le sens externe et interne de l'expérience de la subjectivité dans le contexte mondial des migrations contemporaines. Je remarque en passant ici que le «lien de socialité» des individus au sein des villes-métropoles passe très concrètement, aujourd'hui, par la compréhension de ces vastes mouvements historiques et internationaux qui témoignent de ceci, puisque, à l'échelle locale, le seul moyen de lutter contre la xénophobie, par exemple, est de contextualiser la «rencontre de l'étranger» comme étant inhérente à l'expérience mondiale des deux derniers siècles, et comme étant intrinsèque à la définition même de toutes les sociétés contemporaines qui se mesurent à leur capacité de réfléchir les courants universels à l'œuvre dans le monde ${ }^{29}$. Les expériences individuelles des personnes ayant migré vers les métropoles sont ainsi très exactement à l'image des villes, portant en elles les traces des grands événements historiques du $\mathrm{xx}^{\mathrm{e}}$ siècle. Et c'est dans la fragmentation de cette histoire universelle se répercutant dans les existences individuelles que le tissu métropolitain développe ses particularités locales, qui s'ouvrent ainsi à la reconnaissance de la diversité des expériences sur le fond de la permanence d'une reconnaissance symbolique de l'universalité «mondaine» de cette expérience - que ce soit dans le développement des "ghettos», ou dans le développement de la texture multiculturelle des scènes urbaines. Les villes-métropoles contemporaines sont ainsi devenues avant tout la destination de tous ces «étrangers».

Ici, il va sans dire que l' «étranger» en vient à signifier la part symbolique que l'individu de la masse porte en lui de manière générale, même sans le savoir toujours tout à fait, parce que cette condition est devenue la condition politique universelle première de la personne au sein des sociétés de masse; c'est en effet au niveau premier de la représentation politique, sociétale et individuelle, que la société de masse déploie sa problématique, et c'est sur le plan fondamental de cet anonymat généralisé de la figure du citoyen, ainsi que des possibilités d'identification aux événements du monde qui en découlent, que toutes les recherches personnelles, sociales, culturelles se définissent désormais de manière a priori. Les villes-métropoles réfléchissent alors avant tout ce statut d'étrangeté dans les formes

28. Raymond Williams (1973, p. 279-288) a souligné cette caractéristique fondamentale de l'expérience citadine contemporaine, et cette problématique a plus récemment alimenté notamment le discours du cosmopolitisme du point de vue de la « décolonisation» chez des auteurs comme Homi Bhabha ou James Clifford; voir la critique qu'en fait Pheng Cheah (1999).

29. Voir notamment à ce sujet Kristeva (1991). 
mêmes de leur développement, dans un déploiement horizontal et vertical qui déboussole, littéralement, les sens et la perception, avant de les plier à la logique de leur organisation interne. Et c'est parce qu'elles permettent, mieux que tout autre lieu, la rencontre dialectique de cette diversité que les villes-métropoles en deviennent le symbole. Chacune d'elles offre en effet en condensé la représentation d'une sortie de l'anonymat universel, par sa capacité d'établir une différence significative quant à l'expérience du monde contemporain, alors que simultanément aucune d'elles en particulier ne peut prétendre en épuiser le sens ${ }^{30}$. La logique d'équivalence ne vaut ici précisément que par l'évidence de ce qui ne la rencontre pas tout à fait.

À cet égard, une certaine logique d'organisation interne des métropoles contemporaines tient pour beaucoup, comme on le sait, aux définitions de l'économie politique qui en ont modelé les formes dans leurs phases industrielles, puis postindustrielles, d'une part, et, d'autre part, aux définitions de l'urbanisme qui en ont affirmé les contours dans des phases d'urbanisation, de suburbanisation et de «ré-urbanisation ». Dans son travail de rationalisation de l'espace et du temps, l'économie politique a fait jouer sa propre logique d'équivalence généralisée - qui devient en réalité une logique de différenciation concrète par répartition inégale de ses produits - en permettant une régulation de plus en plus «individualisée » et différemment hiérarchisée au sein de la société de masse ${ }^{31}$. Cela se réfléchit bien entendu dans le phénomène de la division du travail, mais aussi directement par l'évolution des formes de propriété où, en plus de la propriété privée «classique» (i.e. individuelle), ont émergé des formes autres telles la propriété «corporative» (i.e. celle des entreprises privées nationales, puis transnationales), la propriété «sociale» (i.e. celle des services publics et sociaux), ainsi que la propriété « consomptive» (i.e. celle de la consommation à moyen et à long terme). Cette répartition des formes de propriété, à elle seule, s'est ainsi répercutée au sein de l'organisation urbaine, notamment dans la concentration de la propriété « corporative» dans les centre-villes d'un côté, et de la propriété «consomptive» de l'autre, dont les banlieues sont souvent devenues les représentantes exemplaires, alors que la propriété «sociale» définissait l'organisation urbaine plus large. Cette répartition, parfaitement inégale par ailleurs au sein des populations, en est donc également venue à former divers horizons et diverses barrières, voire des frontières symboliques dans l'existence quotidienne, et c'est dans les villes-métropoles que se côtoient ainsi directement les inégalités les plus inimaginables - qui sont alors assez précisément à l’image du monde

30. "The cosmopolitan profile - to be of the moment, to be at the centre - is hard won and constantly under revision. It distinguishes both the exemplary city dweller and the exemplary city, that is, the city which makes such a system of desire compelling. This system of desire identifies the ecstatic relation to the present and to centrality (to this time and to this space) as the sine qua non of universal man, the practice of doing and, so, of being universal, of being universality incarnate. That universality is done in the city makes this a common feature of any significant city: since any city has the capacity to fertilize such postures, universality becomes a recognizable ground for worldliness in any and every place.» (Blum, 2003, p. 136)

31. Ici, «individualisée» ne signifie pas que ce mouvement découle de l'individualisme volontaire, produit de l'autonomie individuelle, mais bien d'un individualisme imposé (par la division du travail et de la consommation notamment), qui s'appréhende mieux en fait en termes d'« isolationnisme». 
contemporain en général ${ }^{32}$. Paradoxalement cependant, la ville-métropole contient en ellemême ces diverses frontières symboliques, faisant de sa propre localisation une détermination spatio-temporelle assumant directement ces frontières (ainsi que leurs traversées, leurs fermetures, leurs transgressions, etc.). L'espace-temps métropolitain contemporain est ainsi devenu le lieu de toutes les frontières (définies auparavant selon leur contour national), et simultanément, le lieu de croisement et de dépassement de toutes les frontières et c'est ce qui fait en partie aussi leur caractère cosmopolitique. D'ailleurs, de Montréal à Mexico, ou de Toronto à Dublin, de New Delhi à Honk Kong ou à Sao Paulo, les tensions qui apparaissent dans les métropoles sont souvent suscitées par les inégalités économiques, qui ont ainsi souvent remplacé la religion ou la culture comme foyer des conflits sociaux, voire comme motivation de la violence générale, puisqu'elles résultent d'une contradiction entre le statut politique formellement égalitaire dont peuvent se réclamer les individus en tant que citoyens, et la répartition concrètement inégalitaire, et surtout parfaitement injuste, de la propriété et des modes de subsistance dans le monde ${ }^{33}$. Dans des conditions qui rendent parfois les citadins d'une même ville si étrangers les uns aux autres qu'ils en deviennent potentiellement des « ennemis » les uns pour les autres, c'est donc toujours en fait la fausse règle d'équivalence de l'économie politique qui joue, à travers la division du travail et la structuration des divers marchés, en même temps que l'organisation urbaine tente la médiation de ces partages des espaces-temps sociaux en mouvement, comme cadre effectif de réalisation de la citoyenneté formelle. La logique de ce développement, qui donne au rythme de la vie urbaine sa cadence accélérée et ses fractures sociales multiples, est ainsi devenue pour nous d'une certaine banalité.

Il y a en effet, simultanément au développement de l'originalité des métropoles comme phénomène urbain contemporain, une banalité dans leur logique d'organisation qui fait que leur " étrangeté » première y paraît tout aussi bien réduite à l'extrême, se confondant alors même tout à fait avec la «familiarité » des villes-métropoles; bien sûr on rencontre des variations dans l'organisation de l'espace urbain entre Toronto et Montréal, entre ces dernières et Berlin ou Amsterdam, et entre toutes celles-ci et New York, Lima ou Santiago, mais également, on reconnaît aisément dans tous ces cas la familiarité

32. Les populations vivant du recyclage des déchets des villes-métropoles, qui s'installent ainsi à demeure tout juste à la frange de l'espace urbain, témoignent éloquemment de ces écarts incroyables; Daniel Castillo Durante écrit : «À Asuncion, la capitale du Paraguay, une ville de presque 700 ooo habitants, 50 ooo familles vivent à Cateura, une très grande étendue de terres qui jouxtent le fleuve Paraguay. C'est là que se trouve le dépotoir de la capitale : des montagnes d'ordures et toutes sortes de déchets montrent à ciel ouvert les différentes vagues de consommation tout au long des années. De nombreuses cheminées permettent au gaz de s'échapper sans pour autant éviter des explosions ici et là. Des milliers de familles - beaucoup de mères célibataires parmi elles $-\mathrm{y}$ vivent et y travaillent. Cateura opère comme une ville dans la ville. Débordé par l'accumulation de détritus, le dépotoir gagne du terrain et tend, dans ses frontières, à se confondre avec la ville elle-même. Une économie interne (très diversifiée) avec toute une hiérarchie de fonctions permet à une bonne partie des habitants de Cateura de vivre du recyclage des ordures.» (Castillo Durante, 2003, p. 14-15).

33. Ce qui ne signifie pas bien sûr que les questions culturelles ou religieuses ne continuent pas d'exister dans leurs tensions inhérentes dont les conséquences, lorsqu'elles sont exacerbées, peuvent justement devenir des entraves au cosmopolitisme, comme en témoignent des exemples tels Sarajevo ou Jérusalem - voir à ce propos Sanguin (1998). Sur cette question et ses « soubassements » à Jérusalem, voir Weizman (2003). 
des formes de la ville-métropole contemporaine, devenue précisément aujourd'hui une situation tellement reconnue qu'elle ne déstabilise à peu près plus personne, et qu'elle peut même ennuyer tout le monde, de Paris à Los Angeles, de Londres à Buenos Aires, ou de Vancouver à Quito. Nous sommes en présence ici de la ville-métropole peuplée d'une étrangeté devenue partout trop familière. Et en dehors des différences locales, cette familiarité nous renvoie à l'universalité de la situation des villes-métropoles.

Ce qui souligne alors la condition universelle des individus, dans leur figure duale d'étrangers-familiers au sein des villes-métropoles, c'est en quelque sorte que leurs trajets respectifs en son sein sont devenus routiniers, banals, une existence dont la figure du «blasé » chez Georg Simmel donnait déjà un aperçu également intéressant (Simmel, 1984, p. 66). L'étranger-familier des métropoles ne nous frappe plus tant il nous ressemble, et cela malgré l'excentricité occasionnelle de son trajet (dont le commuter, traduit malencontreusement par «banlieusard», donne peut-être l'exemple le plus saisissant, justement parce que, lorsqu'on s'y arrête, il nous frappe par sa «familiarité étrange »); il en est ainsi aussi cependant du badaud, du passant, du consommateur ou encore même du «flâneur », comme le décrivait Baudelaire, jusqu'au vagabond, au «clochard», aux «squeegees», et même jusqu'à l'itinérant ou au sans-abri, dont la présence devenue très familière dans le paysage urbain nous indique seulement une étrangeté très relative, à laquelle d'ailleurs nous pouvons tous nous identifier assez facilement, dès lors que l'on pénètre ne serait-ce qu'un peu l'intériorité du trajet biographique qui a mené ces diverses figures à la rue $e^{34}$. Dans ces existences qui sont aussi les nôtres, en effet, nous côtoyons en fait la condition humaine du monde contemporain, réfléchie et même exacerbée par l'expérience métropolitaine des individus divers, qui traduit alors à sa façon l'histoire actuelle dans sa forme universelle, témoin de cette forme du cosmopolitisme qui n'affiche plus en apparence ni d'extériorité à son existence concrète et «immédiate », ni d'utopie à sa réalisation d'ensemble, mais qui en rassemble les contradictions. Si le cosmopolitisme moderne prenait forme dans la figure de l'individu émancipé et rationnellement constitué dans le type du bourgeois, entretenu par son universalité utopique, le cosmopolitisme postmoderne s'affirme plutôt ainsi dans la figure des masses d'individus d'où ressortent, par moments, des expressions typiques en en montrant justement l'extension et les limites; tout, c'est-à-dire toute expérience, doit avoir accès à la représentation dans l'expérience des villes-métropoles, si l'on considère l'universalité réelle et problématique du cosmopolitisme contemporain.

Tout est en effet devenu "intégré » aux masses d'individus des villes-métropoles, et la figure de l'étranger-familier transparaît même dans la personne des touristes, ces nouveaux «barbares-civilisés» qui envahissent, parfois même en hordes, les villes-métropoles afin qu'elles tirent profit de leur présence à la fois rassurante et menaçante (ainsi que de leurs «espèces trébuchantes»). Les villes-métropoles peuvent ainsi considérer qu'elles possèdent encore un attrait, qu'elles sont toujours des lieux qui, en dépit du déracine-

34. Voir entre autres Laberge (2000) ainsi que l'ouvrage classique d'Anderson (1961), où ressortaient déjà la particularité de cette figure dans sa marginalité, qui en faisait un être cosmopolite dans sa situation d' «étranger » dans la cité. 
ment qu'elles promeuvent et qu'elles assument, apparaissent tout autant comme des refuges où l'on peut s'arrêter, sinon à demeure, au moins de manière passagère.

La situation des réfugiés politiques, à cet égard, est également significative du rôle que l'on entend faire jouer aujourd'hui aux villes-métropoles comme symboles de l'hospitalité universelle, puisqu'elle rappelle que, quelle que soit la limite politique qui rejoint l'expression individuelle, il ne peut y avoir de lieu où cette dernière ne peut trouver asile. De là cette idée des villes-refuges, comme repères de cet aspect fondamental et élémentaire du cosmopolitisme tel que l'entend Jacques Derrida (1997); en tant qu'élargissements de l'idée des sanctuaires religieux (églises, temples, etc.) servant de retraite ultime aux persécutés, la ville-métropole devient ainsi le réceptacle d'une humanité respectée dans ses définitions universelles de base. Dans cette définition limite du cosmopolitisme contemporain, qui le renvoie à son existence seulement marginale, comme s'il se réduisait à assurer une condition minimale d'existence et surtout d'expression à sa condition proprement politique, ce sont de nouveau les villes-métropoles qui en garantissent l'incarnation concrète. Je conclurai maintenant brièvement en faisant un retour sur ces nouvelles formes du cosmopolitisme, afin d'en tirer quelques éléments de synthèse nous permettant peutêtre d'en saisir la nature et la structure dans les formes diverses qu'il adopte.

\section{CONCLUSION : NOUVELLES FORMES DU COSMOPOLITISME}

Les nouvelles formes du cosmopolitisme contemporain situent précisément leur originalité dans le fait qu'elles apparaissent être liées non pas à un universalisme abstrait, d'un côté, et, de l'autre, qu'elles ne témoignent pas d'un attachement strict à une culture «locale»; ces nouvelles formes sont donc comprises comme étant médiatrices du global et du local, et c'est la raison pour laquelle les villes-métropoles en deviennent les symboles par excellence. Le cosmopolitisme des villes-métropoles se manifeste ainsi dans la tension entre ces deux extrêmes, tension qui rassemble bon nombre de phénomènes contemporains au sein de l'existence sociale. Et cette tension se révèle dans les formes mêmes qui restent jusqu'à un certain point indéterminées, c'est-à-dire encore ouvertes à leur développement et à leur résolution. On pourrait dire ainsi que c'est par ses caractères «difformes", ou " informes», que le cosmopolitisme se présente aujourd'hui au sein des villes-métropoles, dans le sens actif où ces caractères nous informent de sa réalité active. C'est ce qui fait du cosmopolitisme des villes-métropoles un problème vivant, une dialectique active présentement. Et cette activité se mesure dans la distance prise à l'égard du cosmopolitisme moderne, particulièrement en fait parce que le cosmopolitisme, au lieu d'être cantonné à un horizon utopique ou théologique, devient l'enjeu d'un débat véritablement politique. Les villes-métropoles réfléchissent ce débat dans les termes des expressions contradictoires qui nourrissent le développement du cosmopolitisme contemporain, de manière soit explicite, soit implicite, se jouant de façon consciente ou inconsciente dans les manifestations diverses qui meublent la scène du monde contemporain. Le cosmopolitisme devient ainsi la mesure de ces manifestations; les écarts qui existent, non seulement entre les villesmétropoles contemporaines, mais bien à l'intérieur de chacune d'elles, entre ces diverses formes d'expressions, nous donnent une idée des exigences de médiation cosmopolitique 
qui apparaissent sur divers plans de l'expérience sociale. Ces espaces-temps fracturés des villes-métropoles contemporaines dévoilent alors la tâche de la réflexion cosmopolitique dans le monde d'aujourd'hui.

Dans son troisième "article définitif en vue de la paix perpétuelle», Kant stipulait que: «Le droit cosmopolitique doit se restreindre aux conditions de l'hospitalité universelle» (1991, p. 93). Cette restriction se comprenait chez lui en fonction du fait que les domaines du droit civil (jus civitatis) et du droit «national» (jus gentium) étaient assumés de manière autonome par les États devant se fédérer, à l'aune du droit cosmopolitique (jus cosmopoliticum). Cette «hospitalité universelle» est bien la caractéristique fondamentale des villesmétropoles. Mais que signifie alors aujourd'hui ce «droit cosmopolitique», dans la mesure où les mœurs de nos société ont libéré au sein des métropoles les formes nouvelles du cosmopolitisme auxquelles nous avons fait référence? Prise dans son sens strict, la notion d' «hospitalité » traduisait pour Kant la relation qui unit l'hôte à l'étranger, c'est-à-dire qu'elle « signifie le droit pour l'étranger, à son arrivée sur le territoire d'un autre, de ne pas être traité par lui en ennemi ${ }^{35} »$. On voit sans doute ici, après que les rapports avec la figure de l'étranger (et même celle de l'«ennemi») se furent quelque peu éclaircis au sein de l'expérience des métropoles, toute la distance qui nous sépare de la pensée moderne, en même temps que tout ce qui nous lie à elle; en rapprochant en effet l'étranger du familier, en faisant de l'étranger-familier la figure universelle de la personne au sein des sociétés de masse, la société contemporaine a imposé des nouvelles formes du cosmopolitisme dont les villes-métropoles demeurent les centres et les révélateurs les plus actifs, en rayonnant ainsi à la fois dans l'intériorité subjective individuelle et dans l'extériorité du monde. L'hospitalité des métropoles contemporaines se mesure donc aux formes du cosmopolitisme qu'elles accueillent en elles, mais dans un rapport qui n'est jamais unilatéral, puisqu'il se joue toujours au sein de la tension dialectique de l'identification - différenciation, par le biais du rapport attraction -, répulsion qui devient le cœur de la relation métropolitaine sur laquelle repose la définition nouvelle, et pour ainsi dire radicale, de notre insociable sociabilité; ainsi, c'est l'hostilité généralisée des métropoles qui constitue aujourd'hui le revers exact de leur hospitalité.

La tension entre hostilité et hospitalité des villes-métropoles contemporaines nous donne alors le pouls, la pulsation, de l'existence cosmopolitique contemporaine. C'est dans ce mouvement qu'apparaissent les métropoles comme spectacle du monde. Cela ne signifie pas que le cosmopolitisme y ait atteint sa forme définitive ou achevée, puisque, en effet, cette situation se traduirait par une constitution universelle du monde dans les termes $\mathrm{du}$ droit, définissant alors par le fait même les possibilités d'expression de la justice sociale - et les villes-métropoles nous donnent ainsi un aperçu assez précis du trajet qu'il nous reste à parcourir jusque-là. S'il n'appartient pas aux villes-métropoles d'édicter des lois de citoyenneté, c'est aux villes-métropoles en revanche de montrer quelles sont les mœurs politiques auxquelles ces lois doivent se rattacher. Le spectacle des villes-métropoles révèle en lui-même une teneur cosmopolitique; il resterait aux législations nationales et internationales à la convertir en expérience universelle concrète. Car si le cosmopolitisme doit

35. Kant (1991, p. 93-94). Voir également à ce sujet Chauvier (1996). 
se rendre complètement à ses conditions politiques d'existence, il apparaît également comme mesure de la justice des rapports sociaux qui se déploient en lui. Cela signifie donc également que la reconstitution graduelle du droit civique, du droit national et du droit international est ancrée de manière définitive dans les formes du cosmopolitisme dont nos villes-métropoles nous révèlent en bonne partie la teneur et les enjeux, dans la mesure également où la représentation que l'on en fait s'accorde au spectacle du monde qui nous est ainsi révélé.

\section{RÉSUMÉ}

La définition du cosmopolitisme au sein de la philosophie politique moderne situait son développement dans une perspective à la fois théologique et utopique; par rapport à cela, le développement des villes-métropoles contemporaines a établi des liens directs avec une nouvelle conception du cosmopolitisme. Cette conception est désormais directement liée au phénomène métropolitain dans ce que celui-ci réfléchit les conditions d'existence précises et possibles du cosmopolitisme. L'ordre politique universel qui apparaît ainsi dans les villesmétropoles est cependant traversé de contradictions importantes, qui révèlent les fractures spatio-temporelles présentes dans cette définition du cosmopolitisme contemporain. Ce sont ces tensions, affrontements et contradictions qui doivent toutefois guider l'idée que l'on se fait du cosmopolitisme, puisque c'est là que se donne à voir réellement le spectacle du monde, soit un ensemble de phénomènes qui se manifestent en condensé dans l'expérience des villes. L'article prend la mesure de ces transformations contemporaines du cosmopolitisme à travers la perception des villes-métropoles.

\section{ABSTRACT}

The definition of cosmopolitanism in modern political philosophy has viewed its development from both a theological and utopian perspective. Thus, the development of the contemporary metropolis has established close links with a new conception of cosmopolitanism. This conception is henceforth directly connected with the metropolitan phenomenon insofar as the latter reflects the precise and possible conditions for the existence of cosmopolitanism. However, the universal political order thus emerging in great cities is subject to major contradictions which reveal the spatial and temporal cleavages present in this definition of contemporary cosmopolitanism. But it is these tensions, conflicts and contradictions that must shape one's idea of cosmopolitanism, since it is here that the global spectacle can really be seen, for they represent a set of phenomena that appears in condensed form in the experience of cities. The paper assesses these contemporary transformations of cosmopolitanism through the perception of the metropolis.

\section{RESUMEN}

La definición del cosmopolitismo en la filosofía política moderna situaba su desarrollo en una perspectiva a la vez teológica y utópica; con relación a esto, el desarrollo de las ciudadesmetrópolis contemporáneas estableció vínculos directos con una nueva concepción del cosmopolitismo. Esta concepción está a partir de ahora directamente vinculada al fenómeno metropolitano en cuanto que éste reflexiona las condiciones de existencia precisas y posibles del cosmopolitismo. El orden político universal que aparece así en las ciudades-metrópolis está atravesado sin embargo de contradicciones importantes, que revelan las fracturas espa- 
cio-temporal presentes en esta definición del cosmopolitismo contemporáneo. Son estas tensiones, confrontaciones y contradicciones que deben no obstante guiar la idea que se hace del cosmopolitismo, puesto que es allí que se da a conocer realmente el espectáculo del mundo, o sea un conjunto de fenómenos que se manifiestan en resumen en la experiencia de las ciudades. El artículo toma conciencia de estas transformaciones contemporáneas del cosmopolitismo a través de la percepción de las ciudades-metrópolis.

\section{BIBLIOGRAPHIE}

Abbas, A. (2002), "Cosmopolitan De-Scriptions: Shanghai and Hong Kong», in Breckenridge, C.A., Pollock, S., Bhabha, H.K., Chakrabarty, D., eds., Cosmopolitanism, Durham, Duke University Press, p. 209-228.

Anderson, N. (1961), The Hobo, Chicago, University of Chicago Press.

Beck, U. (2003), Pouvoir et contre-pouvoir à l’ère de la mondialisation, trad. A. Duthoo, Paris, Flammarion.

BelL, D. (1978) The Cultural Contradictions of Capitalism, New York, Basic Books.

Bender, T. (2002), The Unfinished City. New York and the Metropolitan Idea, New York, New Press.

Berger, P.L. (1977), « New York City 1976 : A Signal of Transcendence», dans Facing Up to Modernity : Excursions in Society, Politics, and Religion, New York, Basic Books, p. 211-220.

Blum, A. (2003), The Imaginative Structure of the City, Montréal \& Kingston, McGill-Queen's University Press.

Bourne, R. (1964), «Transnational America», in Carl Reseck, ed., War and the Intellectuals. Essays by Randolph Bourne, 1915-1919, New York, Harper \& Row, p. 107-123.

Bradbury, M. (1983), «The Cities of Modernism», in Bradbury, M., McFarlane, J., eds., Modernism, New York, Penguin Books.

Breckenridge, C.A., Pollock, S., Bhabha, H.K., Chakrabarty, D., eds. (2002), Cosmopolitanism, Durham, Duke University Press.

Caglar, A. (2002), «Media Corporatism and Cosmopolitanism », in Vertovec, S., Cohen, R., eds., Conceiving Cosmopolitanism. Theory, Context, and Practice, Oxford, Oxford University Press, p. 180-190.

Caron, A. (1999), «La restauration de la Vieille-Havane et l'identité cubaine », dans Morisset, L.K., Noppen, L., St-Jacques, D. (dir.), Ville-imaginaire. Ville identitaire. Écho de Québec, Québec, Nota Bene, p. 155176.

Castillo Durante, D. (2003), «Cultures ultrapériphériques, mondialisation et paroles littéraires », dans Castillo Durante, D., Imbert, P., dir., L'interculturel au coeur des Amériques, Ottawa, Presses de l'Université d'Ottawa, 2003, p. 13-20.

Certeau, M. de, Giard, L., Mayol, P. (1994), L'invention du quotidien. 2. Habiter, cuisiner, Paris, Gallimard.

Chauvier, S. (1996) Du droit d'être étranger. Essai sur le concept kantien d'un droit cosmopolitique, Paris, L'Harmattan.

CHeAh, P. (1999), « Given Culture: Rethinking Cosmopolitcal Freedom in Transnationalism », in CHeAH, P., Roвbins, B., eds., Cosmopolitics. Thinking and Feeling Beyond the Nation, Minneapolis, University of Minnesota Press, p. 290-328.

Côté, J.F. (2003), Le triangle d'Hermès. Poe, Stein, Warhol, figures de la modernité esthétique, Bruxelles, La lettre volée.

Coulanges, F. De (1984), La cité antique, Paris, Flammarion, (édition originale 1864).

Derrida, J. (1997), Cosmopolites de tous les pays, encore un effort!, Paris, Galilée.

Dion, R. (1999), «L'imaginaire de Berlin détruit», dans Morisset, L.K., Noppen, L., St-Jacques, D. (dir.), Ville-imaginaire. Ville identitaire. Écho de Québec, Québec, Nota Bene, p. 327-342.

Ferrarotti, F. (1995), «Civil Society as a Polyarchic Form : The City », dans Philip Kasinitz, ed., Metropolis. Center and Symbol of Our times, New York, New York University Press, p. 450-468. 
FreitaG, M. (1971), « De la ville-société à la ville-milieu. L'unité du processus social de constitution et de dissolution de l'objet urbain ", Sociologie et sociétés, vol. $3, \mathrm{n}^{\circ}{ }^{\circ}$, p. $25-57$

Frisby, D. (2001), Cityscapes of Modernity, Cambridge, Polity Press.

García Canclini, N. dir. (1998), Cultura y communicacion en la ciudad de Mexico, 2 vol., Mexico, Grijalbo.

GrésILlon, B. (1998), «Berlin métropole culturelle. Les mutations de la géographie culturelle berlinoise depuis la réunification ", Géographie et culture, n 27, p. 99-114.

Grezner, E. (2001), «Setting the Stage for a New Germany », Public, 22/23, p. 219-242.

Grezner, E. (2002), «The Topographies of Memory in Berlin: the Neue Wache and the Memorial for the Murdered Jews of Europe », Canadian Journal of Urban Research, 11, 1, p. 93-110.

Habermas, J. (1993), L'espace public, trad. M.B. de Launay, Paris, Payot.

Habermas, J. (2000), Après L'État-nation. Une nouvelle constellation politique, trad. R. Rochlitz, Paris, Fayard.

Harvey, D. (1992), The Condition of Postmodernity, Cambridge, Blackwell.

Hegel, G.W.F. (réédition de 1998), Principes de la philosophie du droit, trad. J.F. Kervégan, Paris, PUF.

JАСОВ, L. (2003), «Les interventions artistiques en milieu urbain et la question du cosmopolitisme», dans Daniel Latouche, dir., Voulez-vous manger avec moi? Pratiques interculturelles en France et au Québec, Montréal, Fides, p. 35-47.

JacoBs, J. (1969), The Economy of Cities, New York, Random House.

Jameson, F. (1991), Postmodernism, or The Cultural Logic of Late Capitalism, Durham, Duke University Press. JASPERs, K. (1954) Origine et sens de l'histoire, trad. H. Naef, Paris, Plon.

Kant, E. (réédition de 1991), «Vers la paix perpétuelle», trad. J.F. Poirier, F. Proust, dans Emmanuel Kant, Vers la paix perpétuelle. Que signifie s'orienter dans la pensée. Qu'est-ce que les Lumières?, Paris, Flammarion, p. 73-131.

Kant, E. (réédition de 1985), «Idée d'une histoire universelle d'un point de vue cosmopolitique », dans Critique de la faculté de juger, dir. F. Alquí, Paris, Gallimard, p. 477-495.

KristeVA, J. (1991), Étrangers à nous-mêmes, Paris, Gallimard.

Laberge, D., dir. (2000), L'errance urbaine, Sainte-Foy, Éditions MultiMondes.

Mumford, L. (1940), The Culture of Cities, London, Secker \& Warburg.

Pforte, D. (1991), «Promotion de la culture et politique culturelle», dans Friedrich Lach, Hans-Herbert

S. RäKEL, Berlin à Montréal, Montréal, VLB éditeur, p. 55-73.

Roвin, R. (2001), Berlin Chantiers, Paris, Stock.

SANGuin, A.L. (1998), "Sarajevo avant et après le siège, les mutations culturelles d'une capitale pluriethnique ", Géographie et cultures, nº 27, p. 41-62.

SAssen, S. (1998), "Cities in the Global Economy », in John Knechtel, ed., Alphabet City, 6, p. 172-176.

Sennett, R. (1974), The Fall of Public Man, New York, Vintage Books.

Sennett, R. (2002) «Cosmopolitanism and the Social Experience of Cities», dans Steven Vertovec, Robin Cohen, eds., Conceiving Cosmopolitanism. Theory, Context and Practice, Oxford, Oxford University Press, p. 42-47.

Sсотт, A. J., SojA, E.W. eds. (1998), The City. Los Angeles and Urban Theory at the End of the Twentieth Century, Berkeley, University of California Press.

Seltzer, M. (2003), «Berlin 2000 : "The Image of and Empty Place”, dans Resina, J.R., Ingenschay, D., eds., After-Images of the City, Ithaca, Cornell University Press, p. 61-74.

Simmel, G. (1984), «Métropoles et mentalité », trad. Y. Grafmeyer, dans Joseph, I. (dir.), L'École de Chicago. Naissance de l'écologie urbaine, Paris, Aubier.

SojA, E.W. (2000), Post-Metropolis. Critical Studies of Cities and Regions, Oxford, Blackwell Publishers.

Spengler, O. (1948), Le déclin de l'Occident, 2 vol., trad. M. Tazerout, Paris, Gallimard.

Sutcliffe, A., ed., (1984) Metropolis 1890-1940, Chicago, University of Chicago Press.

Vertovec, S., Cohen, R., eds. (2002), Conceiving Cosmopolitanism. Theory, Context, and Practice, Oxford, Oxford University Press.

Weber, M. (1982), La ville, trad. P. Fritsch, Paris, Aubier. 
Weizman, E. (2003), "The Politics of Verticality: the West Bank as an Architectural Construction », dans Klaus Bisenbach, ed., Territories, Berlin, Institute for Contemporary Art, p. 65-117.

Williams, R. (1973), The Country and the City, New York, Oxford University Press.

Zubaida, S. (2002), « Middle Easter Experiences of Cosmopolitanism », in Vertovec, S., Cohen, R., eds., Conceiving Cosmopoitanism. Theory, Context and Practice, Oxford, Oxford University Press, p. 32-41.

Zukin, S. (1995), The Culture of Cities, Cambridge, Blackwell. 